\title{
(704) Interamnia: a transitional object between a dwarf planet and a typical irregular-shaped minor body $\star, \star \star$
}

\author{
J. Hanuš ${ }^{1}$, P. Vernazza ${ }^{2}$, M. Viikinkoski ${ }^{3}$, M. Ferrais ${ }^{4,2}$, N. Rambaux ${ }^{5}$, E. Podlewska-Gaca ${ }^{6}$, A. Drouard ${ }^{2}$, L. Jorda ${ }^{2}$, \\ E. Jehin ${ }^{4}$, B. Carry ${ }^{7}$, M. Marsset ${ }^{8}$, F. Marchis ${ }^{9}$, B. Warner ${ }^{10}$, R. Behrend ${ }^{11}$, V. Asenjo ${ }^{12}$, N. Berger ${ }^{13}$, \\ M. Bronikowska ${ }^{14}$, T. Brothers ${ }^{8}$, S. Charbonnel ${ }^{15}$, C. Colazo ${ }^{16}$, J.-F. Coliac ${ }^{17}$, R. Duffard ${ }^{18}$, A. Jones ${ }^{19}$, A. Leroy ${ }^{20}$, \\ A. Marciniak ${ }^{6}$, R. Melia ${ }^{16}$, D. Molina ${ }^{21}$, J. Nadolny ${ }^{22}$, M. Person ${ }^{8}$, O. Pejcha ${ }^{23}$, H. Riemis ${ }^{24}$, B. Shappee ${ }^{25}$, \\ K. Sobkowiak ${ }^{6}$, F. Soldán ${ }^{26}$, D. Suys ${ }^{24}$, R. Szakats ${ }^{27}$, J. Vantomme ${ }^{24}$, M. Birlan ${ }^{5}$, J. Berthier ${ }^{5}$, P. Bartczak ${ }^{6}$, \\ C. Dumas ${ }^{28}$, G. Dudziński ${ }^{6}$, J. Durech ${ }^{1}$, J. Castillo-Rogez ${ }^{29}$, F. Cipriani ${ }^{30}$, R. Fetick ${ }^{2}$, T. Fusco ${ }^{2}$, J. Grice ${ }^{7,31}$, \\ M. Kaasalainen ${ }^{3}$, A. Kryszczynska ${ }^{6}$, P. Lamy ${ }^{32}$, T. Michalowski ${ }^{6}$, P. Michel ${ }^{7}$, T. Santana-Ros ${ }^{33,34}$, P. Tanga ${ }^{7}$, \\ F. Vachier ${ }^{5}$, A. Vigan ${ }^{2}$, O. Witasse ${ }^{30}$, and B. Yang ${ }^{35}$
}

(Affiliations can be found after the references)

Received 5 September 2019 / Accepted 21 November 2019

\begin{abstract}
Context. With an estimated diameter in the $320-350 \mathrm{~km}$ range, (704) Interamnia is the fifth largest main belt asteroid and one of the few bodies that fills the gap in size between the four largest bodies with $D>400 \mathrm{~km}$ (Ceres, Vesta, Pallas and Hygiea) and the numerous smaller bodies with diameter $\leq 200 \mathrm{~km}$. However, despite its large size, little is known about the shape and spin state of Interamnia and, therefore, about its bulk composition and past collisional evolution.

Aims. We aimed to test at what size and mass the shape of a small body departs from a nearly ellipsoidal equilibrium shape (as observed in the case of the four largest asteroids) to an irregular shape as routinely observed in the case of smaller $(D \leq 200 \mathrm{~km})$ bodies.

Methods. We observed Interamnia as part of our ESO VLT/SPHERE large program (ID: 199.C-0074) at thirteen different epochs. In addition, several new optical lightcurves were recorded. These data, along with stellar occultation data from the literature, were fed to the All-Data Asteroid Modeling algorithm to reconstruct the 3D-shape model of Interamnia and to determine its spin state.

Results. Interamnia's volume-equivalent diameter of $332 \pm 6 \mathrm{~km}$ implies a bulk density of $\rho=1.98 \pm 0.68 \mathrm{~g} \mathrm{~cm}^{-3}$, which suggests that Interamnia - like Ceres and Hygiea - contains a high fraction of water ice, consistent with the paucity of apparent craters. Our observations reveal a shape that can be well approximated by an ellipsoid, and that is compatible with a fluid hydrostatic equilibrium at the $2 \sigma$ level.

Conclusions. The rather regular shape of Interamnia implies that the size and mass limit, under which the shapes of minor bodies with a high amount of water ice in the subsurface become irregular, has to be searched among smaller $(D \leq 300 \mathrm{~km})$ less massive $\left(m \leq 3 \times 10^{19} \mathrm{~kg}\right)$ bodies.
\end{abstract}

Key words. minor planets, asteroids: individual: (704) Interamnia - methods: observational - techniques: high angular resolution techniques: photometric

\section{Introduction}

Because of their large masses, Solar-System bodies with diameters larger than $\sim 900 \mathrm{~km}$ possess rounded, ellipsoidal shapes, consistent with hydrostatic equilibrium. On the other side of the mass range, very small bodies (diameters $\leq 100 \mathrm{~km}$ ) tend to possess highly irregular shapes, with the notable exception of some $D \leq 5 \mathrm{~km}$ bodies that are affected by the socalled YORP effect (Yarkovsky-O'Keefe-Radzievskii-Paddack, Rubincam 2000; Vokrouhlický et al. 2003), and which have similar shapes to a spinning top (e.g., Ryugu, or Bennu, Watanabe et al. 2019; Nolan et al. 2013). The theory of the hydrostatic equilibrium of homogeneous bodies is well established

\footnotetext{
* The reduced images are only available at the CDS via anonymous ftp to cdsarc.u-strasbg. fr (130.79.128.5) or via http://cdsarc. u-strasbg.fr/viz-bin/cat/J/A+A/633/A65

$\star \star$ Based on observations made with ESO Telescopes at the La Silla Paranal Observatory under program 199.C-0074 (PI: Vernazza).
}

(e.g., Chandrasekhar 1969), whereas for differentiated bodies, approaches based on Clairaut equations (e.g., Dermott 1979; Chambat et al. 2010; Rambaux et al. 2015, 2017) or the numerical non-perturbative method are still under developement (Hubbard 2013). From an observational point of view, it remains to be tested at what size range the shape of a typical minor body transits from a nearly rounded equilibrium shape to an irregular shape and to what extent this size range depends on factors such as the bulk composition of the minor planet or its collisional and thermal history.

Investigating these questions is one of the main motivations of our European Southern Observatory (ESO) large program (id: 199.C-0074; Vernazza et al. 2018) of which the aim is to constrain the shape of the forty largest main-belt asteroids. So far, our program has revealed that (10) Hygiea, the fourth largest main-belt asteroid ( $\mathrm{D} \sim 434 \mathrm{~km}$ ) possesses a shape that is nearly as spherical as that of (1) Ceres (Vernazza et al. 2019), while being twice as small, whereas $D \sim 100-200 \mathrm{~km}$ bodies 
[(89) Julia, (16) Psyche, (41) Daphne] possess irregular shapes (Vernazza et al. 2018; Viikinkoski et al. 2018; Carry et al. 2019). Asteroid (7) Iris $(D \sim 214 \mathrm{~km})$ is an intermediate case, as its shape appears to be consistent with that of an oblate spheroid with a large equatorial excavation (Hanuš et al. 2019).

Asteroid (704) Interamnia, the fifth largest body in the main belt with a volume equivalent diameter in the $320-350 \mathrm{~km}$ size range (Drummond et al. 2009; Masiero et al. 2014), is one of the very few asteroids that fills the gap in size between Hygiea and $D \sim 250 \mathrm{~km}$-sized bodies. The remaining main belt asteroids in this size range are (31) Euphrosyne $(D=282 \pm 10 \mathrm{~km}$, Masiero et al. 2013), (52) Europa $(D=314 \pm 5 \mathrm{~km}$, Hanuš et al. 2017), (65) Cybele $(D=296 \pm 25 \mathrm{~km}$, Viikinkoski et al. 2017), and (511) Davida ( $D=311 \pm 5 \mathrm{~km}$, Viikinkoski et al. 2017). Shapes of Europa and Davida already show some departures from a rotational triaxial ellipsoid (Conrad et al. 2007; Merline et al. 2013). Interamnia thus appears as another key target for investigating at what size the shape of a small body becomes irregular.

So far, little is known about Interamnia. It lacks a dynamical family, implying that it avoided a giant impact over the last $\sim 3$ Gyrs. It was classified in the B-spectral class following the Bus taxonomy based on visible data alone, whereas it is labeled a C-type in the Bus-DeMeo taxonomy (Clark et al. 2010). In the visible and near-infrared spectral range, it thus appears similar to objects such as (1) Ceres, (10) Hygiea, (24) Themis, and (52) Europa, which have been connected to interplanetary dust particles (IDPs) rather than to carbonaceous chondrites (Vernazza et al. 2015, 2017; Marsset et al. 2016). Interamnia is also of great interest to the present study, being the largest asteroid for which a detailed shape model (convex or with local topography) and consistent spin-state solutions do not yet exist. This may be due to its shape being rather spherical, as suggested by the small brightness variations in its lightcurves (Tempesti 1975; Warner 2018) and by the Keck disk-resolved images obtained by Drummond \& Christou (2008).

Here, we present high-angular resolution imaging observations of Interamnia with ESO VLT/SPHERE/ZIMPOL that were performed as part of our large program. We use these observations along with several newly acquired lightcurves to constrain its 3D shape and its spin for the first time.

\section{Observations}

The observations used in our analysis of physical properties of Interamnia consist of disk-resolved images from the VLT telescope and disk-integrated optical lightcurves from various sources, including our observing campaign.

\subsection{Disk-resolved data}

Interamnia was observed with VLT/SPHERE/ZIMPOL (Spectro-Polarimetric High-contrast Exoplanet REsearch, Zurich IMaging POLarimeter, Thalmann et al. 2008) in the narrow band imaging configuration $\left(N \_R\right.$ filter; filter central wavelength $=645.9 \mathrm{~nm}$, width $=56.7 \mathrm{~nm}$ ) during two consecutive apparitions in August-September 2017, and between December 2018 and January 2019. During both apparitions, the angular size of Interamnia was in the $0.20-0.26^{\prime \prime}$ range, with a slightly larger angular size during the first apparition. Interamnia's extent on the images reaches up to 80 pixels. Both datasets sample the whole rotation phase of Interamnia, although not as evenly as initially expected (we recall here that our nominal observing strategy is to image our large program targets every 60 degrees throughout their rotation). Nonetheless, the satisfactory rotation phase coverage, along with a nearly equator-on geometry during both apparitions lead to a nearly complete surface coverage $(\sim 95 \%)$ that makes it possible to constrain the three dimensions of Interamnia well.

The reduced images were deconvolved with the Mistral algorithm (see Fétick et al. 2019, for details about the deconvolution procedure) and are shown in Figs. A.1 and A.2. Table A.1 contains full information about the data.

Finally, in addition to the AO data, we also utilized four stellar occultations obtained in 1996, 2003, 2007, and 2012. However, the stellar occultations are largely redundant given the coverage of our SPHERE observations. We provide details about these observations in Table A.2.

\subsection{Optical photometry}

We compiled a large dataset of 189 optical lightcurves sampling 15 apparitions. These data include lightcurves downloaded from the Asteroid Photometric Catalog (APC, Piironen et al. 2001) with original references: Tempesti (1975); Lustig \& Hahn (1976); Shevchenko et al. (1992); Michalowski et al. (1995). Many lightcurves were also provided through the courbes de rotation d'astéroïdes et de comètes database $\left(\mathrm{CdR}^{1}\right)$, maintained by Raoul Behrend at the Observatoire de Genève, and through the $\mathrm{ALCDEF}^{2}$ database maintained by Brian Warner (Warner 2018). The largest photometric dataset was obtained from the SuperWASP archive (Grice et al. 2017): 114 lightcurves spanning years 2006-2011. Finally, one lightcurve was obtained at Wallace Observatory, and a densely covered dataset was obtained by the TRAPPIST-South and -North (Jehin et al. 2011) as a support for this study.

Additional photometric data were gathered within the observing campaign of the "Small Bodies: Near And Far" project (Müller et al. 2018), with partial participation of GaiaGOSA observers. Gaia-GOSA ${ }^{3}$ is a web service dedicated to amateur observers willing to support asteroid studies through targeted photometric campaigns. The website makes it possible to coordinate a worldwide observing campaign, which is especially important for slow rotating objects requiring long-term observations over several nights. Interamnia was observed on Gaia-GOSA during its last two apparitions (2017 and 2018), providing new lightcurves for our dataset.

Finally, we also made use of sparsely sampled $V$-band photometry from the All-Sky Automated Survey for Supernovae (ASAS-SN, Shappee et al. 2014; Kochanek et al. 2017) and Gaia Data Release 2 (DR2, Gaia Collaboration 2018). The ASAS-SN data sample five consequent apparitions between 2013 and 2018, and contain 196 individually calibrated measurements in the Johnson $V$ band. Gaia DR2 data are internally calibrated, however, they are also limited to only 16 individual measurements. The sparse data are processed following the same procedures applied, for example, in Hanuš et al. (2011), or Ďurech \& Hanuš (2018). Other sparsely sampled data used so far for the shape modeling (e.g., USNO-Flagstaff, Catalina Sky Survey, Lowell, Ďrech et al. 2009, 2016; Hanuš et al. 2011, 2013) have photometric uncertainties, at best, comparable to the lightcurve amplitude of Interamnia (usually $<0.1 \mathrm{mag}$ ), which makes them useless for the shape modeling. On the other hand, the high-photometric precision of the ASAS-SN data $(\sim 0.04 \mathrm{mag})$ and Gaia DR2 ( $\sim 0.02 \mathrm{mag})$ implies that brightness changes due to irregular

\footnotetext{
http://obswww. unige.ch/ behrend/page_cou.html

http://alcdef.org/

www.gaiagosa.eu
} 


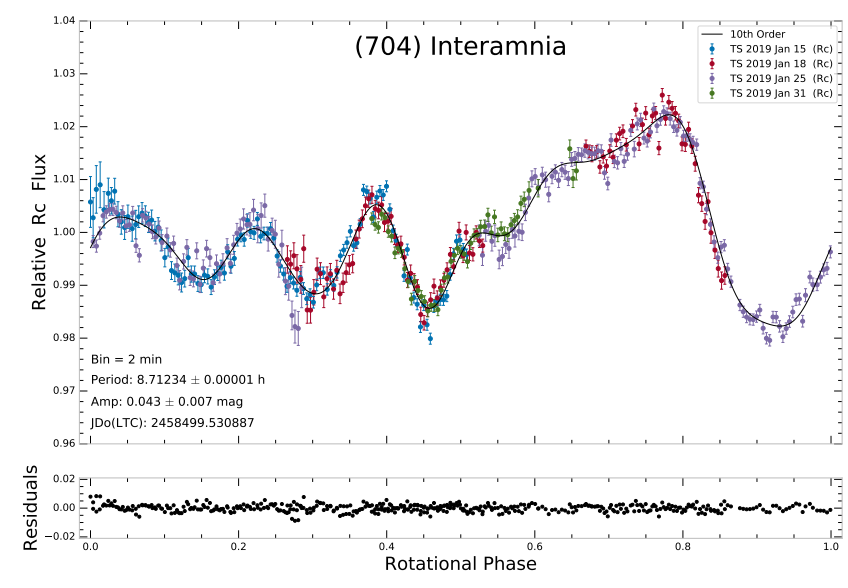

Fig. 1. Upper panel: composite lightcurve of (704) Interamnia obtained with TRAPPIST-South telescope. A Fourier series of tenth order is fitted to the data. Lower panel: residuals of the fit.

shape and spin state are distinguishable from the photometric noise. Sparse data are particularly useful for the spin-state determination, because they cover a large range of observing geometries (i.e., phase angles).

The basic characteristics of the photometric data are listed in Table A.3. In general, Interamnia's lightcurves exhibit a brightness variation pattern consistent with a synodic rotation period of $\sim 8.7 \mathrm{~h}$ and rather small amplitude of the brightness changes within the rotation (usually $<0.1 \mathrm{mag}$, see, e.g., Fig. 1). These small changes make the determination of a unique shape model and spin-state solution challenging.

\section{Results}

The rich datasets of disk-integrated optical lightcurves and VLT/SPHERE/ZIMPOL disk-resolved images enabled us to derive the convex shape model of Interamnia, as well as its 3D-shape model with local topography. Moreover, we also estimated Interamnia's bulk density and analysed its shape with respect to the hydrostatic equilibrium. Finally, we discuss few identified surface features.

\subsection{Spin-state determination by convex inversion}

To derive the first reliable shape and spin-state solution for Interamnia, we implemented the standard convex inversion method of Kaasalainen \& Torppa (2001) and Kaasalainen et al. (2001) that takes disk-integrated data as the only data input, and searches the set of parameters describing the shape and rotation state that best match the data. The search was done on a grid of parameters, where each set of input parameters converges to a local minimum in the parameter space. We tested all reasonable combinations of relevant parameters to find those that correspond to the global minimum. In convex inversion, the shape is parametrized by a convex polyhedron, the rotation state is described by the sidereal rotation period, and the ecliptic coordinates (longitude and latitude) of the spin axis, and we used a simple three-parameter phase function relation that is necessary when sparse data are included (Kaasalainen et al. 2001). The convex inversion procedure essentially consists of two parts. Firstly, we ran the convex inversion for rotation periods from the 8.6-8.8 h interval, which contains all previous estimates that concentrate near $\sim 8.72 \mathrm{~h}$. The step in the rotation period was selected in a way that each local minimum is sampled (Kaasalainen et al. 2001). For each period, we ran the convex inversion with 10 different initial pole orientations isotropically distributed on a sphere and selected only the best fitting solution. Then, we constructed the dependence of the rms value on the sampled period in Fig. A.3. This periodogram has a clear minimum near $8.71 \mathrm{~h}$, moreover, only one period value $(P=8.71236 \mathrm{~h})$ provides a significantly better fit to the observed data than all the other periods. We applied the same criteria as in Hanuš et al. (2018, for more details and additional references) to distinguish between acceptable solutions and those that should already be rejected. Secondly, we ran the convex inversion with the unique period found in the previous step, with a higher shape model resolution, and for many pole orientations $(\sim 50)$ isotropically distributed on a sphere. Only four pole solutions fell within the rms limit from Hanuš et al. (2018). Moreover, two solutions out of the four with the worst fit had non-physical shapes with their maximum moment of inertia significantly nonaligned with the rotation axis. Therefore, we derived only two possible spin-state and shape solutions, which we list in Table 1.

Our spin-state solutions are rather different from those previously published with the main disagreement in the ecliptic latitude - we found a prograde rotation with ecliptic latitude of $\sim 40-60^{\circ}$, while previous authors derived mostly smaller values between $-20^{\circ}$ and $20^{\circ}$ (Michalowski 1993; Michalowski et al. 1995; De Angelis 1995; Drummond \& Christou 2008), or even $-50^{\circ}$ (Satō et al. 2014) for the latitude. On the other hand, the determinations for the ecliptic longitude are mostly consistent with each other. The closest solution to ours is from Drummond et al. (2009) based on disk-resolved images from Keck. The rather significant differences are likely caused by (i) Interamnia having small brightness variations, and (ii) the fact that the spin-state determination based on photometric data with low signal-to-noise ratio is challenging and could lead to inaccurate determinations (or to underestimated uncertainties, Marciniak et al. 2015). Our first reliable spin-state solution of Interamnia is used as an input for the shape modeling with All-Data Asteroid Modeling (ADAM) in the following section.

\subsection{D-shape reconstruction with $A D A M$}

The disk-resolved data allow us to perform the 3D-shape optimization with the ADAM algorithm. Firstly, we compared the SPHERE images with corresponding projections of the two shape solutions derived by the convex inversion and found that only one solution is consistent with the images (see Table. 1), therefore, we continued the shape modeling only with the preferred rotation state solution as an input.

We proceeded with the modeling the standard way (see, e.g., Viikinkoski et al. 2018; Hanuš et al. 2019): we constructed a low-resolution shape model using the octantoid (Viikinkoski et al. 2015a) shape parametrization while balancing the fit to the optical lightcurves, SPHERE images, and stellar occultations. We applied the ADAM algorithm to a dataset of 189 optical lightcurves, $60 \mathrm{VLT} / \mathrm{SPHERE/ZIMPOL}$ images, and four stellar occultations. Then, we increased the shape model resolution and the weight of the SPHERE data with respect to the lightcurves and occultations, and used the low-resolution shape model as an initial input. We tested several combinations of shape resolutions and relative weighting of the observed data to confirm the consistency of our results. The comparison between the shape model projections and SPHERE observations is shown in Fig. 2. Moreover, we also provide the model fit to the stellar occultations in Fig. 3. All four stellar occultations agree well with our shape model. 
Table 1. Summary of published spin-state solutions for Interamnia.

\begin{tabular}{cccccl}
\hline \hline $\begin{array}{c}\lambda_{1} \\
(\mathrm{deg})\end{array}$ & $\begin{array}{c}\beta_{1} \\
(\mathrm{deg})\end{array}$ & $\begin{array}{c}\lambda_{2} \\
(\mathrm{deg})\end{array}$ & $\begin{array}{c}\beta_{2} \\
(\mathrm{deg})\end{array}$ & $\begin{array}{c}P \\
(\mathrm{~h})\end{array}$ & Note \\
\hline $43 \pm 8$ & $-21 \pm 9$ & $224 \pm 10$ & $-22 \pm 10$ & - & Michalowski (1993) \\
$47 \pm 10$ & $-3 \pm 10$ & $227 \pm 10$ & $1 \pm 10$ & - & De Angelis (1995) \\
$51 \pm 15$ & $22 \pm 10$ & & & - & Michalowski et al. (1995) \\
$36 \pm 5$ & $12 \pm 5$ & & & $8.72729 \pm 0.00001$ & Drummond \& Christou (2008) \\
$47 \pm 10$ & $66 \pm 10$ & & & - & Drummond et al. (2009) \\
$259 \pm 8$ & $-50 \pm 5$ & & & $8.712355 \pm 0.000005$ & Satō et al. (2014) \\
$87 \pm 10$ & $63 \pm 10$ & $226 \pm 10$ & $43 \pm 10$ & $8.71234 \pm 0.00001$ & This work, CI \\
$87 \pm 5$ & $62 \pm 5$ & & & This work, ADAM \\
\hline
\end{tabular}

Notes. The table gives the ecliptic longitude $\lambda$ and latitude $\beta$ of all possible pole solutions with their uncertainties, the sidereal rotation period $P$, and the reference. Our second pole solution from convex inversion (CI) has been rejected due to inconsistency with the SPHERE images. ${ }^{(a)}$ Such uncertainty in the rotation period is unrealistic.

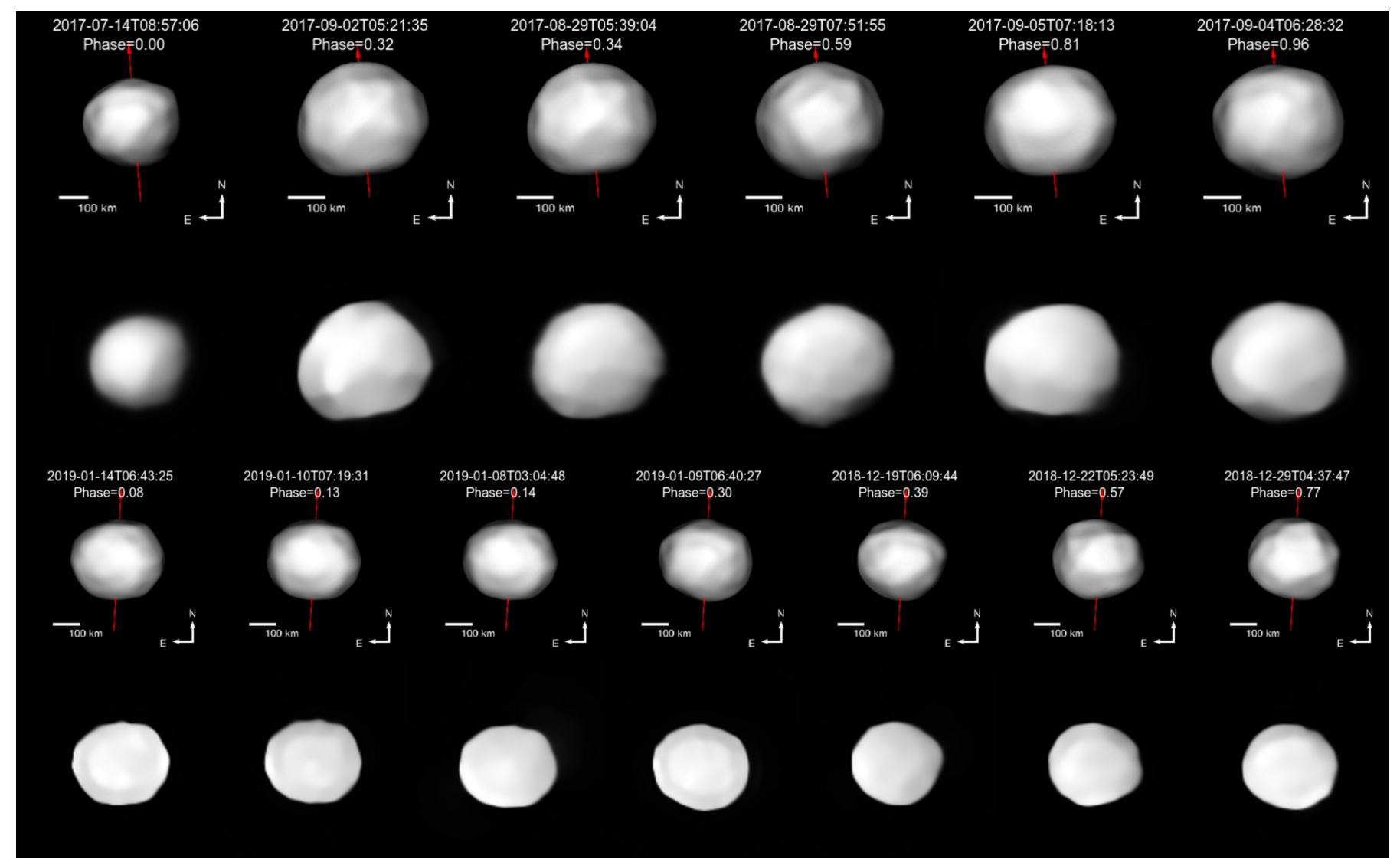

Fig. 2. Comparison between VLT/SPHERE/ZIMPOL deconvolved images of Interamnia (second and fourth rows) and the corresponding projections of our ADAM shape model (first and third row). The red line indicates the position of the rotation axis. We use a nonrealistic illumination to highlight the local topography of the model.

The physical properties of our final solution are listed in Table 2. The uncertainties reflect the typical ranges of parameters within the various individual solutions we obtained (for different shape resolutions, relative data weights). The uncertainties are also consistent with the size of one to two pixels. The volume-equivalent diameter of Interamnia $(332 \pm 6 \mathrm{~km})$ is well constrained because of the equator-on observations during both apparitions, and because the overall coverage of the AO observations amounts to $\sim 95 \%$ of the model surface area. Moreover, for the same reason, the $c$ dimension is also reliably estimated, which happens rather rarely, because the usually limited geometry coverage of the images makes the determination of the $c$ dimension inaccurate. Our size estimate is larger than those of Drummond et al. (2009) and Satō et al. (2014), but both are in agreement with ours within the $1 \sigma$ uncertainties.

The shape model along with the lightcurve data and the fit to all datasets have been uploaded to the online Database of Asteroid Models from Inversion Techniques (DAMIT ${ }^{4}$, Durech et al. 2010).

\footnotetext{
4 http://astro.troja.mff.cuni.cz/projects/damit
} 

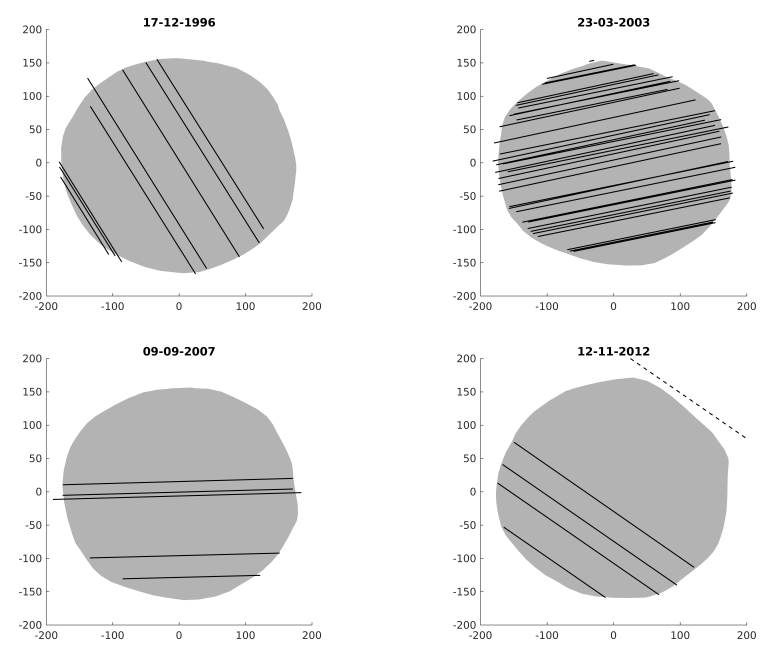

Fig. 3. Observed occultation chords and model silhouettes. The dashed line is a negative observation. North is up and east to the left. The axis scale corresponds to kilometers.

\subsection{Density}

We combined the derived volume of Interamnia with the best estimate of its mass $(3.79 \pm 1.28) \times 10^{19} \mathrm{~kg}$ (Table A.4 and Fig. A.4) and obtained a bulk density estimate of $1.98 \pm 0.68 \mathrm{~g} \mathrm{~cm}^{-3}$. Our mass estimate is based on all relevant determinations collected in the literature (as we did in our previous studies, for instance, Carry et al. 2012; Vernazza et al. 2018; Viikinkoski et al. 2018; Hanuš et al. 2019).

The relative uncertainty affecting the bulk density of Interamnia exceeds $30 \%$, preventing us from drawing meaningful conclusions about the body's composition. For instance, the bulk density is compatible within $1 \sigma$ error with those of the two largest C-type asteroids, Ceres $\left(2.161 \pm 0.003 \mathrm{~g} \mathrm{~cm}^{-3}\right.$, Park et al. 2019) and Hygiea $\left(1.94 \pm 0.25 \mathrm{~g} \mathrm{~cm}^{-3}\right.$, Vernazza et al. 2019), but also of silicate bodies such as (25143) Itokawa $\left(1.90 \pm 0.13 \mathrm{~g} \mathrm{~cm}^{-3}\right.$, Fujiwara et al. 2006) or (433) Eros (2.67 $\pm 0.10 \mathrm{~g} \mathrm{~cm}^{-3}$, Veverka et al. 2000). Current estimates of the densities of asteroids with masses greater than $\sim 5 \times 10^{18} \mathrm{~kg}$ imply a small amount of macroporosity within these bodies (Carry 2012; Viikinkoski et al. 2015b; Marsset et al. 2017; Carry et al. 2019; Hanuš et al. 2019), and spectroscopic observations of Interamnia in the 3-micron region have revealed the presence of hydrated material at its surface (Usui et al. 2019) and spectral similarity to Ceres (Rivkin et al. 2019). Therefore, we can assume that Interamnia's bulk density is close to that of Ceres. This implies a large amount of water inside Interamnia, likely as a mixture of ice and phyllosilicates, as in the case of Ceres.

\subsection{Shape analysis}

As a first step, we performed an analysis of Interamnia's shape, similar to the one performed in the case of Hygiea (Vernazza et al. 2019). We fitted Interamnia's 3D-shape model with an ellipsoid and subsequently measured the radial difference between the two shapes. It appears that the large-scale topography of Interamnia is very subdued, without noticeable large impact basins on its surface (Fig. A.5), similar to that of Ceres and Hygiea (Vernazza et al. 2019). As in the case of Hygiea, the relative volume difference between Interamnia's 3D-shape model and that of its best-fitting ellipsoid is $0.2 \%$, which implies that Interamnia's shape is very close to that of an ellipsoid. Next, we calculated the sphericity of Interamnia as done previously in the case of Hygiea (Vernazza et al. 2019). We found a sphericity value of 0.9880 , similar to that of Vesta (0.9860), and only marginally lower than that of the nearly spherical dwarf planet candidate Hygiea (0.9975, Vernazza et al. 2019).

Given the ellipsoidal shape of Interamnia and the fact that its $a$ and $b$ axes have similar lengths (within errors) and that the $c$ dimension is shorter than the $a$ and $b$ axes, we investigated whether Interamnia's shape may be at hydrostatic equilibrium. We investigated both (i) a homogeneous and (ii) a core-mantle differentiated case. Indeed, given the large uncertainty of the bulk density coming from the large mass uncertainty, both models are viable possibilities.

The hydrostatic equilibrium figure of an homogeneous body can be computed using MacLaurin's equation (e.g., Chandrasekhar 1969):

$\frac{\Omega^{2}}{\pi G \rho}=\frac{2 \sqrt{1-e^{2}}}{e^{3}}\left(3-2 e^{2}\right) \arcsin (e)-6 \frac{1-e^{2}}{e^{2}}$,

where $G$ is the gravitational constant, $\Omega$ is the rotational velocity, and $e$ is the ellipticity of the body shape defined by

$e^{2}=1-\left(\frac{c}{a}\right)^{2}$.

The MacLaurin equation is not valid for a differentiated body (Moritz 1990). In this case, the hydrostatic equilibrium figure can be derived through Clairaut's equations developed to an order that depends on a parameter $m$ called geodetic parameter (Chambat et al. 2010; Rambaux et al. 2015):

$m=\frac{\Omega^{2} R^{3}}{G M}$,

where $\Omega$ is the angular spin velocity, $R$ the mean radius, and $M$ the mass of the body. Considering the particular value of $m$ and the quality of available observations, Clairaut's equations may be developed to first, second, or third order (Lanzano 1974). For example, at first order, Clairaut's equation is written as (Kopal 1960; Lanzano 1974)

$r^{2} \ddot{f}_{2}+6 \gamma r \dot{f}_{2}+6(\gamma-1) f_{2}=0$,

where $f_{2}$ corresponds to the coefficient of the Legendre polynomial of degree two of the equipotential surface $s$

$s(r, \theta)=r\left(1+f_{2}(r) P_{2}(\cos \theta)\right)$,

and $\gamma=\rho(r) / \bar{\rho}(r)$ is the ratio between the density of the layer at $r$ and the mean density at $r$. Lanzano (1974) developed the equations up to order three by introducing the following coefficients:

$s(r, \theta)=r\left(1+f_{2}(r) P_{2}(\cos \theta)+f_{4}(r) P_{4}(\cos \theta)+f_{6}(r) P_{6}(\cos \theta)\right)$,

and he obtained a system of three differential equations and boundary conditions (see the equations in Lanzano 1974). A numerical scheme to solve these equations has previously been applied to the hydrostatic figures of Earth (Chambat et al. 2010), Ceres (Rambaux et al. 2015; Park et al. 2016) and Pallas (Marsset et al., in prep.), and now to a differentiated Interamnia.

For the homogeneous case, we computed $a-c$ values for mean densities within the $1300-2700 \mathrm{~kg} \mathrm{~m}^{-3}$ range, while for the core-mantle differentiated model, we explored the 1100$1600 \mathrm{~kg} \mathrm{~m}^{-3}$ range for the mantle density and the 
Table 2. Volume-equivalent diameter $(D)$, dimensions along the major axis $(a, b, c)$, sidereal rotation period $(P)$, spin-axis ecliptic $\mathbf{J} 2000$ coordinates (longitude $\lambda$ and latitude $\beta$ ), mass $(m)$, and bulk density $(\rho)$ of Interamnia as determined here, compared with the work of Drummond \& Christou (2008); Drummond et al. (2009); Satō et al. (2014).

\begin{tabular}{llcccc}
\hline \hline Parameter & Unit & Drummond \& Christou (2008) & Drummond et al. (2009) & Satō et al. (2014) & This work, ADAM \\
\hline$D$ & $\mathrm{~km}$ & & $319 \pm 9$ & $327 \pm 3$ & $332 \pm 6$ \\
$\lambda$ & $\mathrm{deg}$. & $36 \pm 6$ & $47 \pm 3$ & $259 \pm 8$ & $87 \pm 5$ \\
$\beta$ & $\mathrm{deg}$. & $12 \pm 11$ & $66 \pm 3$ & $-50 \pm 5$ & $62 \pm 5$ \\
$P$ & $\mathrm{~h}$ & $385 \pm 53$ & 8.727 & $8.728967167(7)$ & $8.712336(10)$ \\
$a$ & $\mathrm{~km}$ & $337 \pm 21$ & $349 \pm 4$ & $362 \pm 3$ & $362 \pm 8$ \\
$b$ & $\mathrm{~km}$ & $163 \pm 184$ & $339 \pm 3$ & $324 \pm 5$ & $348 \pm 8$ \\
$c$ & $\mathrm{~km}$ & $1.14 \pm 0.17$ & $1.03 \pm 0.01$ & $297 \pm 4$ & $310 \pm 8$ \\
$a / b$ & & $1.24 \pm 0.10$ & $1.15 \pm 0.02$ & $1.04 \pm 0.02$ \\
$b / c$ & & & $6.20 \pm 0.01$ & $1.13 \pm 0.02$ \\
$m$ & $10^{19} \mathrm{~kg}$ & & & $3.8 \pm 1.0$ & $3.79 \pm 1.28$ \\
$\rho$ & $\mathrm{g} \mathrm{cm}^{-3}$ & & & & $1.98 \pm 0.68$ \\
\hline
\end{tabular}

Notes. Uncertainties correspond to $1 \sigma$ values.

References. ${ }^{(a)}$ Michalak (2001).

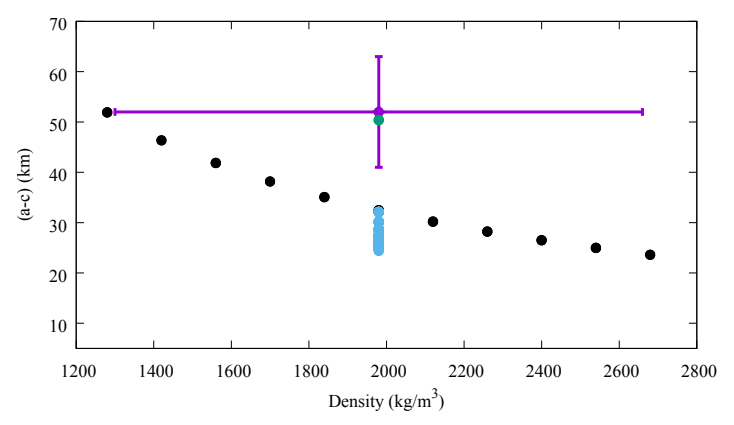

Fig. 4. Results of $(a-c)$ for Interamnia as a function of mean density for homogeneous case (sequence of black dots) and core-mantle differentiated case (blue vertical dots). For the latter case, we explored the $1100-1600 \mathrm{~kg} \mathrm{~m}^{-3}$ range for the mantle density, and the 2050$3400 \mathrm{~kg} \mathrm{~m}^{-3}$ range for the core density while keeping the bulk density at a constant value of $1980 \mathrm{~kg} \mathrm{~m}^{-3}$. The value of $a-c$ decreases when mass increases with depth. The purple cross represents the value derived in Sect. 3.4 with its $1 \sigma$ uncertainty (uncertainties of $a$ and $b$ are added quadratically). Finally, the green dot represents the values of $(a-c)$ for the homogeneous case and Interamnia's assumed faster rotation period of $7.1 \mathrm{~h}$.

2050-3400 $\mathrm{kg} \mathrm{m}^{-3}$ range for the core density while keeping the bulk density at $1980 \mathrm{~kg} \mathrm{~m}^{-3}$. As expected, $a-c$ decreases when mass increases with depth. In Fig. 4, we present the $a-c$ values as a function of mean density for both the homogeneous case (black dots) and the core-mantle differentiated case (blue dots) and compare them with the observed values.

Assuming Interamnia's bulk density of $2000 \mathrm{~kg} \mathrm{~m}^{-3}$ (i.e., similar to that of Ceres or Hygiea), its shape is consistent with hydrostatic equilibrium at the $2 \sigma$ level. We further calculated, by aiming for the central value of $a-c$, that Interamnia's shape would be at hydrostatic equilibrium at the $1 \sigma$ level for a slightly shorter rotation period (7.1 h, see Fig. 4), assuming homogeneous interior. The core-mantle differentiated case requires slightly larger despinning. This is in agreement with the collisional models that predict statistical preference of despinning by impacts (Ševeček et al. 2019).

Overall, these results are compatible with a formation of Interamnia at hydrostatic equilibrium. Interamnia's global equilibrium shape is likely a consequence of both its large mass and the initial presence of a large amount of water ice in its interior. During its early history, a large fraction of the water ice would have melted due to the radioactive decay of ${ }^{26} \mathrm{Al}$ implying the presence of liquid water in its interior, and thus an early fluid interior as is the case for Vesta, Ceres, and Hygiea (Takir \& Emery 2012; Vernazza et al. 2017).

\subsection{Surface topography}

We observe only two large depressions (apparent dark regions) in the bottom-right parts of the images with rotation phases 0.32 and 0.96 (first apparition). In addition, a few mountainlike features can be observed in the object's contours. The most prominent one lies very close to the north pole and is visible at three epochs from the second apparition (rotation phases 0.08 , 0.13 and 0.14 ). This feature could be a central peak of a $\sim 150$ $200 \mathrm{~km}$ large crater. Two similar topographic features are located to the bottom right of the image at rotation phase 0.77 (second apparition), and on the right of rotation phase 0.57 (Fig. 5).

Compared with the large topographic variations found on S-type asteroids such as (3) Juno, (6) Hebe, and (7) Iris (Viikinkoski et al. 2015b; Marsset et al. 2017; Hanuš et al. 2019), Interamnia's surface appears relatively smooth with only a few basins or depressions. From this point of view, Interamnia appears very similar to Hygiea and Ceres (Vernazza et al. 2019). A plausible explanation for the lack of obvious craters at the resolution of these SPHERE images may be, as proposed in the case of Hygiea (Vernazza et al. 2019), that the craters are mostly complex flat-floored rather than simple bowl-shaped. The expected simple-to-complex crater transition diameter for Interamnia, assuming a water-rich composition for its mantle in agreement with our density estimate, should be around $30 \mathrm{~km}$ (Hiesinger et al. 2016) (the transition diameter for a rock-dominated composition would be around $70 \mathrm{~km}$ ). Given the spatial resolution of our observations $(D \sim 30-40 \mathrm{~km})$, the paucity of large bowl-shaped craters on Interamnia can be attributed to its water-rich mantle composition.

\section{Summary}

We derived the first reliable spin-state solution of Interamnia. This success was only possible due to a large participation in 


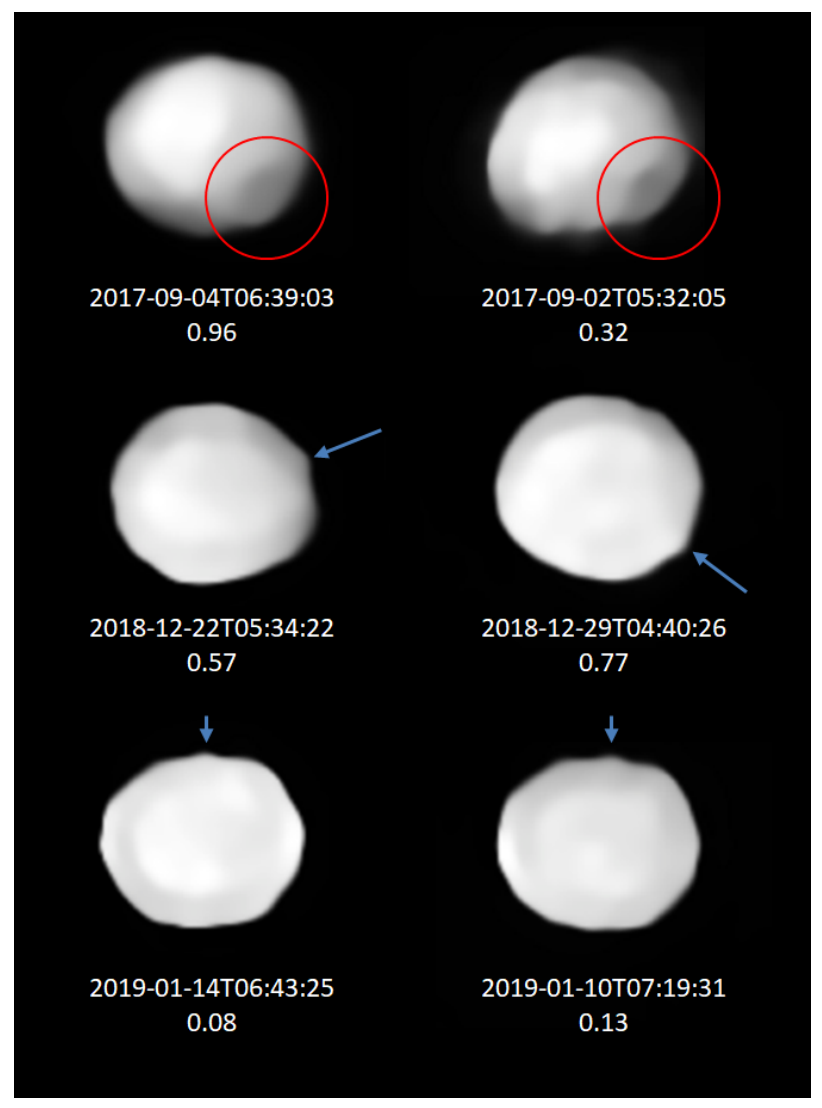

Fig. 5. Topographic features identified on Interamnia. The arrows indicate potential surface features (central peaks of large impact basins) and the red circles the two darker circular regions.

our photometric campaign and data mining from survey telescopes (SuperWASP, ASAS-SN, Gaia). The role of observers with small aperture telescopes was essential.

Our 3D-shape model of Interamnia derived by ADAM from the spectacular SPHERE disk-resolved images is nearly ellipsoidal with almost equal equatorial dimensions $(a / b=1.04)$ and is only slightly flattened with $b / c=1.13$. Interamnia's shape appears to be in hydrostatic equilibrium at the $2 \sigma$ level. It follows that the size and mass limit under which minor bodies' shapes become irregular has to be searched among smaller $(D \leq 300 \mathrm{~km})$ less massive $\left(m \leq 3 \times 10^{19} \mathrm{~kg}\right)$ bodies.

Our volume equivalent diameter of $332 \pm 6 \mathrm{~km}$ makes Interamnia the fifth largest object in the main belt after (1) Ceres, (2) Pallas, (4) Vesta, and (10) Hygiea. The other two 300-kmclass bodies - (52) Europa and (65) Cybele - are likely smaller than Interamnia, although their size estimates have rather large uncertainties. Finally, spectroscopic observations in the near infrared and the bulk density of $\rho=1.98 \pm 0.68 \mathrm{~g} \mathrm{~cm}^{-3}$ suggests that Interamnia - like Ceres and Hygiea - contains a high fraction of water in the subsurface. This would provide a natural explanation for the lack of obvious craters wider than a few tens of $\mathrm{km}$, as well as for its ellipsoidal/regular shape, similarly to what has been proposed for Hygiea by Vernazza et al. (2019).

Acknowledgements. This work has been supported by the Czech Science Foundation through grant 18-09470S (J.H., J.D.) and by the Charles University Research program No. UNCE/SCI/023. This research was supported by INTEREXCELLENCE grant LTAUSA18093 from the Czech Ministry of Education, Youth, and Sports (J.H. and O.P.). The research of O.P. is additionally supported by Horizon 2020 ERC Starting Grant "Cat-In-hAT" (grant agreement \#803158) and award PRIMUS/SCI/17 from Charles University. P.V., A.D., and B.C. were supported by CNRS/INSU/PNP. M.M. was supported by the National Aeronautics and Space Administration under Grant No. 80NSSC18K0849 issued through the Planetary Astronomy Program. This work was supported by the National Science Centre, Poland, through grant no. 2014/13/D/ST9/01818 (A.M.). The research leading to these results has received funding from the European Union's Horizon 2020 Research and Innovation Programme, under Grant Agreement no 687378 (SBNAF). This project has been supported by the GINOP-2.3.2-152016-00003 and NKFIH K125015 grants of the Hungarian National Research, Development and Innovation Office (NKFIH) and by the Lendület grant LP201231 of the Hungarian Academy of Sciences. TRAPPIST-North is a project funded by the University of Liège, in collaboration with Cadi Ayyad University of Marrakech (Morocco). TRAPPIST-South is a project funded by the Belgian FNRS under grant FRFC 2.5.594.09. F.E.J. is a FNRS Senior Research Associate. ASAS-SN thanks the Las Cumbres Observatory and its staff for its continuing support of the ASAS-SN project. ASAS-SN is supported by the Gordon and Betty Moore Foundation through grant GBMF5490 to the Ohio State University and NSF grant AST-1515927. Development of ASAS-SN has been supported by NSF grant AST-0908816, the Mt. Cuba Astronomical Foundation, the Center for Cosmology and AstroParticle Physics at the Ohio State University, the Chinese Academy of Sciences South America Center for Astronomy (CASSACA), the Villum Foundation, and George Skestos.

\section{References}

Baer, J., \& Chesley, S. R. 2008, Celest. Mech. Dyn. Astron., 100, 27 Baer, J., \& Chesley, S. R. 2017, AJ, 154, 76

Baer, J., Chesley, S. R., \& Matson, R. D. 2011, AJ, 141, 143

Carry, B. 2012, Planet. Space Sci., 73, 98

Carry, B., Kaasalainen, M., Merline, W. J., et al. 2012, Planet. Space Sci., 66, 200 Carry, B., Vachier, F., Berthier, J., et al. 2019, A\&A, 623, A132

Chambat, F., Ricard, Y., \& Valette, B. 2010, Geophys. J. Int., 183, 727

Chandrasekhar, S. 1969, Ellipsoidal Figures of Equilibrium (New Haven, USA: Yale University Press)

Chernetenko, Y. A., \& Kochetova, O. M. 2002, ESA SP, 500, 437

Clark, B. E., Ziffer, J., Nesvorny, D., et al. 2010, J. Geophys. Res. Planets, 115, E06005

Conrad, A. R., Dumas, C., Merline, W. J., et al. 2007, Icarus, 191, 616

De Angelis, G. 1995, Planet. Space Sci., 43, 649

Dermott, S. F. 1979, Icarus, 37, 575

Drummond, J., \& Christou, J. 2008, Icarus, 197, 480

Drummond, J., Christou, J., \& Nelson, J. 2009, Icarus, 202, 147

Ďurech, J., \& Hanuš, J. 2018, A\&A, 620, A91

Durech, J., Kaasalainen, M., Warner, B. D., et al. 2009, A\&A, 493, 291

Durech, J., Sidorin, V., \& Kaasalainen, M. 2010, A\&A, 513, A46

Durech, J., Hanuš, J., Oszkiewicz, D., \& Vančo, R. 2016, A\&A, 587, A48

Fétick, R. J., Jorda, L., Vernazza, P., et al. 2019, A\&A, 623, A6

Fienga, A., Laskar, J., Morley, T., et al. 2009, A\&A, 507, 1675

Fienga, A., Kuchynka, P., Laskar, J., Manche, H., \& Gastineau, M. 2011, EPSC-DPS Joint Meeting 2011, 1879

Fienga, A., Manche, H., Laskar, J., Gastineau, M., \& Verma, A. 2013, ArXiv e-prints [arXiv:1301.1510]

Fienga, A., Manche, H., Laskar, J., Gastineau, M., \& Verma, A. 2014, ArXiv e-prints [arXiv:1405.0484]

Folkner, W. M., Williams, J. G., \& Boggs, D. H. 2009, IPN Prog. Rep., 42, 1

Fujiwara, A., Kawaguchi, J., Yeomans, D. K., et al. 2006, Science, 312, 1330

Gaia Collaboration (Spoto, F., et al.) 2018, A\&A, 616, A13

Goffin, E. 2014, A\&A, 565, A56

Grice, J., Snodgrass, C., Green, S. F., Parley, N. R., \& Carry, B. 2017, in Asteroids, Comets, and Meteors: ACM 2017 (Mankato, USA: Capstone Publishers) Hanuš, J., Ďurech, J., Brož, M., et al. 2011, A\&A, 530, A134

Hanuš, J., Durech, J., Brož, M., et al. 2013, A\&A, 551, A67

Hanuš, J., Viikinkoski, M., Marchis, F., et al. 2017, A\&A, 601, A114

Hanuš, J., Vokrouhlický, D., Delbo', M., et al. 2018, A\&A, 620, L8

Hanuš, J., Marsset, M., Vernazza, P., et al. 2019, A\&A, 624, A121

Hiesinger, H., Marchi, S., Schmedemann, N., et al. 2016, Science, 353, 4758

Hubbard, W. B. 2013, ApJ, 768, 43

Ivantsov, A. 2008, Planet. Space Sci., 56, 1857

Jehin, E., Gillon, M., Queloz, D., et al. 2011, The Messenger, 145, 2

Kaasalainen, M., \& Torppa, J. 2001, Icarus, 153, 24

Kaasalainen, M., Torppa, J., \& Muinonen, K. 2001, Icarus, 153, 37

Kochanek, C. S., Shappee, B. J., Stanek, K. Z., et al. 2017, PASP, 129, 104502

Kochetova, O. M. 2004, Sol. Syst. Res., 38, 66

Kochetova, O. M., \& Chernetenko, Y. A. 2014, Sol. Sys. Res., 48, 295

Konopliv, A. S., Asmar, S. W., Folkner, W. M., et al. 2011, Icarus, 211, 401

Kopal, S. 1960, Figures of Equilibrium of Celestial Bodies (Madison, USA: University of Wisconsin Press) 
Krasinsky, G. A., Pitjeva, E. V., Vasiliev, M. V., \& Yagudina, E. I. 2001, Commun. IAA RAS, 6, 114

Kuchynka, P., \& Folkner, W. M. 2013, Icarus, 222, 243

Landgraf, W. 1992, IAU Symp., 152, 179

Lanzano, P. 1974, Ap\&SS, 29, 161

Lustig, G., \& Hahn, G. 1976, Acta Phys. Austriaca, 44, 199

Marciniak, A., Pilcher, F., Oszkiewicz, D., et al. 2015, Planet. Space Sci., 118, 256

Marsset, M., Vernazza, P., Birlan, M., et al. 2016, A\&A, 586, A15

Marsset, M., Carry, B., Dumas, C., et al. 2017, A\&A, 604, A64

Masiero, J. R., Mainzer, A. K., Bauer, J. M., et al. 2013, ApJ, 770, 7

Masiero, J. R., Grav, T., Mainzer, A. K., et al. 2014, ApJ, 791, 121

Merline, W. J., Drummond, J. D., Carry, B., et al. 2013, Icarus, 225, 794

Michalak, G. 2001, A\&A, 374, 703

Michalowski, T. 1993, Icarus, 106, 563

Michalowski, T., Velichko, F. P., Di Martino, M., et al. 1995, Icarus, 118, 292

Moritz, H. 1990, The Figure of the Earth : Theoretical Geodesy and the Earth's Interior (Heidelberg, Germany: Wichmann)

Müller, T. G., Marciniak, A., Kiss, C., et al. 2018, Adv. Space Res., 62, 2326

Nolan, M. C., Magri, C., Howell, E. S., et al. 2013, Icarus, 226, 629

Park, R., Konopliv, A., Bills, B., et al. 2016, EGU General Assembly Conf. Abstracts, 18, 8395

Park, R. S., Vaughan, A. T., Konopliv, A. S., et al. 2019, Icarus, 319, 812

Piironen, J., Lagerkvist, C., Torppa, J., Kaasalainen, M., \& Warner, B. 2001, BAAS, 33, 1562

Pitjeva, E. V. 2013, Sol. Syst. Res., 47, 386

Rambaux, N., Chambat, F., \& Castillo-Rogez, J. C. 2015, A\&A, 584, A127

Rambaux, N., Baguet, D., Chambat, F., \& Castillo-Rogez, J. C. 2017, ApJ, 850, L9

Rivkin, A. S., Howell, E. S., \& Emery, J. P. 2019, J Geophys. Res. Planets, 124, 1393

Rubincam, D. P. 2000, Icarus, 148, 2

Satō, I., Buie, M., Maley, P. D., et al. 2014, Int. J. Astron. Astrophys., 4, 91

Ševeček, P., Brož, M., \& Jutzi, M. 2019, A\&A, 629, A122

Shappee, B. J., Prieto, J. L., Grupe, D., et al. 2014, ApJ, 788, 48

Shevchenko, V. G., Chernyi, V. G., Kruglyi, I. N., et al. 1992, Icarus, 100, 295

Siltala, L., \& Granvik, M. 2017, Icarus, 297, 149

Somenzi, L., Fienga, A., Laskar, J., \& Kuchynka, P. 2010, Planet. Space Sci., 58, 858

Takir, D., \& Emery, J. P. 2012, Icarus, 219, 641

Tempesti, P. 1975, Mem. Soc. Astron. It., 46, 397

Thalmann, C., Schmid, H. M., Boccaletti, A., et al. 2008, Proc. SPIE, 7014, $70143 \mathrm{~F}$

Usui, F., Hasegawa, S., Ootsubo, T., \& Onaka, T. 2019, PASJ, 71, 1

Vasiliev, M. V., \& Yagudina, E. I. 1999, Commun. IAA RAS, 4, 98

Vernazza, P., Marsset, M., Beck, P., et al. 2015, ApJ, 806, 204

Vernazza, P., Castillo-Rogez, J., Beck, P., et al. 2017, AJ, 153, 72

Vernazza, P., Brož, M., Drouard, A., et al. 2018, A\&A, 618, A154

Vernazza, P., Jorda, L., Ševeček, P., et al. 2019, Nat. Astron., in press, http: //doi.org/10.1038/s41550-019-0915-8

Veverka, J., Robinson, M., Thomas, P., et al. 2000, Meteorit. Planet. Sci., 35, 164

Viikinkoski, M., Kaasalainen, M., \& Ďurech, J. 2015a, A\&A, 576, A8

Viikinkoski, M., Kaasalainen, M., Durech, J., et al. 2015b, A\&A, 581, L3

Viikinkoski, M., Hanuš, J., Kaasalainen, M., Marchis, F., \& Durech, J. 2017, A\&A, 607, A117

Viikinkoski, M., Vernazza, P., Hanuš, J., et al. 2018, A\&A, 619, L3

Viswanathan, V., Fienga, A., Gastineau, M., \& Laskar, J. 2017, Notes Scientifiques et Techniques de l'Institut de mécanique céleste, (ISSN 1621-3823), 108, 39

Vokrouhlický, D., Nesvorný, D., \& Bottke, W. F. 2003, Nature, 425, 147

Warner, B. D. 2018, Minor Planet Bull., 45, 39

Watanabe, S., Hirabayashi, M., Hirata, N., et al. 2019, Science, 364, 268

Yang, X.-Y., Zhang, Y.-Y., \& Li, X.-Q. 1965, Acta Astron. Sin., 13, 66

Zielenbach, W. 2011, AJ, 142, 120

${ }^{1}$ Institute of Astronomy, Faculty of Mathematics and Physics, Charles University, V Holešovičkách 2, 18000 Prague, Czech Republic e-mail: pepa@sirrah.troja.mff.cuni.cz
2 Aix Marseille Univ, CNRS, LAM, Laboratoire d'Astrophysique de Marseille, Marseille, France

3 Mathematics and Statistics, Tampere University, 33720 Tampere, Finland

4 Space sciences, Technologies and Astrophysics Research Institute, Université de Liège, Allée du 6 Août 17, 4000 Liège, Belgium

${ }^{5}$ IMCCE, Observatoire de Paris, 77 avenue Denfert-Rochereau, 75014 Paris Cedex, France

${ }^{6}$ Astronomical Observatory Institute, Faculty of Physics, Adam Mickiewicz University, ul. Słoneczna 36, 60-286 Poznań, Poland

7 Université Côte d'Azur, Observatoire de la Côte d'Azur, CNRS, Laboratoire Lagrange, France

${ }^{8}$ Department of Earth, Atmospheric and Planetary Sciences, MIT, 77 Massachusetts Avenue, Cambridge, MA 02139, USA

9 SETI Institute, Carl Sagan Center, 189 Bernado Avenue, Mountain View CA 94043, USA

${ }^{10}$ Center for Solar System Studies, 446 Sycamore Ave., Eaton, CO 80615, USA

11 Geneva Observatory, 1290 Sauverny, Switzerland

12 Asociación Astronómica Astro Henares, Centro de Recursos Asociativos El Cerro C/ Manuel Azaña, 28823 Coslada, Madrid, Spain

13490 chemin du Gonnet, 38440 Saint Jean de Bournay, France

${ }^{14}$ Institute of Geology, A. Mickiewicz University, Krygowskiego 12, 61-606 Poznań, Poland

15 Observatoire de Durtal, 49430 Durtal, France

16 Observatorio Astronómico de Córdoba, Córdoba, Argentina

1720 parc des Pervenches, 13012 Marseille, France

18 Instituto de Astrofisica de Andalucia - CSIC. Glorieta de la Astronomía s/n, 18008 Granada, Spain

19 I64, SL6 1XE Maidenhead, UK

${ }^{20}$ Uranoscope, Avenue Carnot 7, 77220 Gretz-Armainvilliers, France

21 Anunaki Observatory, Calle de los Llanos, 28410 Manzanares el Real, Spain

22 Departamento de Astrofisica, Universidad de La Laguna, $38206 \mathrm{La}$ Laguna, Tenerife, Spain

${ }^{23}$ Institute of Theoretical Physics, Faculty of Mathematics and Physics, Charles University, V Holešovičkách 2, 18000 Prague, Czech Republic

${ }^{24}$ Courbes de rotation d'astéroïdes et de comètes, CdR, Geneva Observatory, 1290 Sauverny, Switzerland

25 Institute for Astronomy, University of Hawai'i, 2680 Woodlawn Drive, Honolulu, HI 96822, USA

${ }^{26}$ Observatorio Amanecer de Arrakis, Alcalá de Guadaíra, Sevilla, Spain

${ }^{27}$ Konkoly Observatory, Research Centre for Astronomy and Earth Sciences, Hungarian Academy of Sciences, Konkoly Thege 15-17, 1121 Budapest, Hungary

28 Thirty-Meter-Telescope, 100 West Walnut St, Suite 300, Pasadena, CA 91124, USA

29 Jet Propulsion Laboratory, California Institute of Technology, 4800 Oak Grove Drive, Pasadena, CA 91109, USA

30 European Space Agency, ESTEC - Scientific Support Office, Keplerlaan 1, Noordwijk 2200 AG, The Netherlands

31 Open University, School of Physical Sciences, The Open University, MK7 6AA, UK

${ }^{32}$ Laboratoire Atmosphères, Milieux et Observations Spatiales, CNRS \& UVSQY, Guyancourt, France

33 Departamento de Física, Ingeniería de Sistemas y Teoría de la Señal, Universidad de Alicante, 03080 Alicante, Spain

${ }^{34}$ Institut de Ciències del Cosmos, Universitat de Barcelona (IEECUB), Martí i Franquès 1, 08028 Barcelona, Spain

35 European Southern Observatory (ESO), Alonso de Cordova 3107, 1900 Casilla Vitacura, Santiago, Chile 


\section{Appendix A: Additional figures and tables}

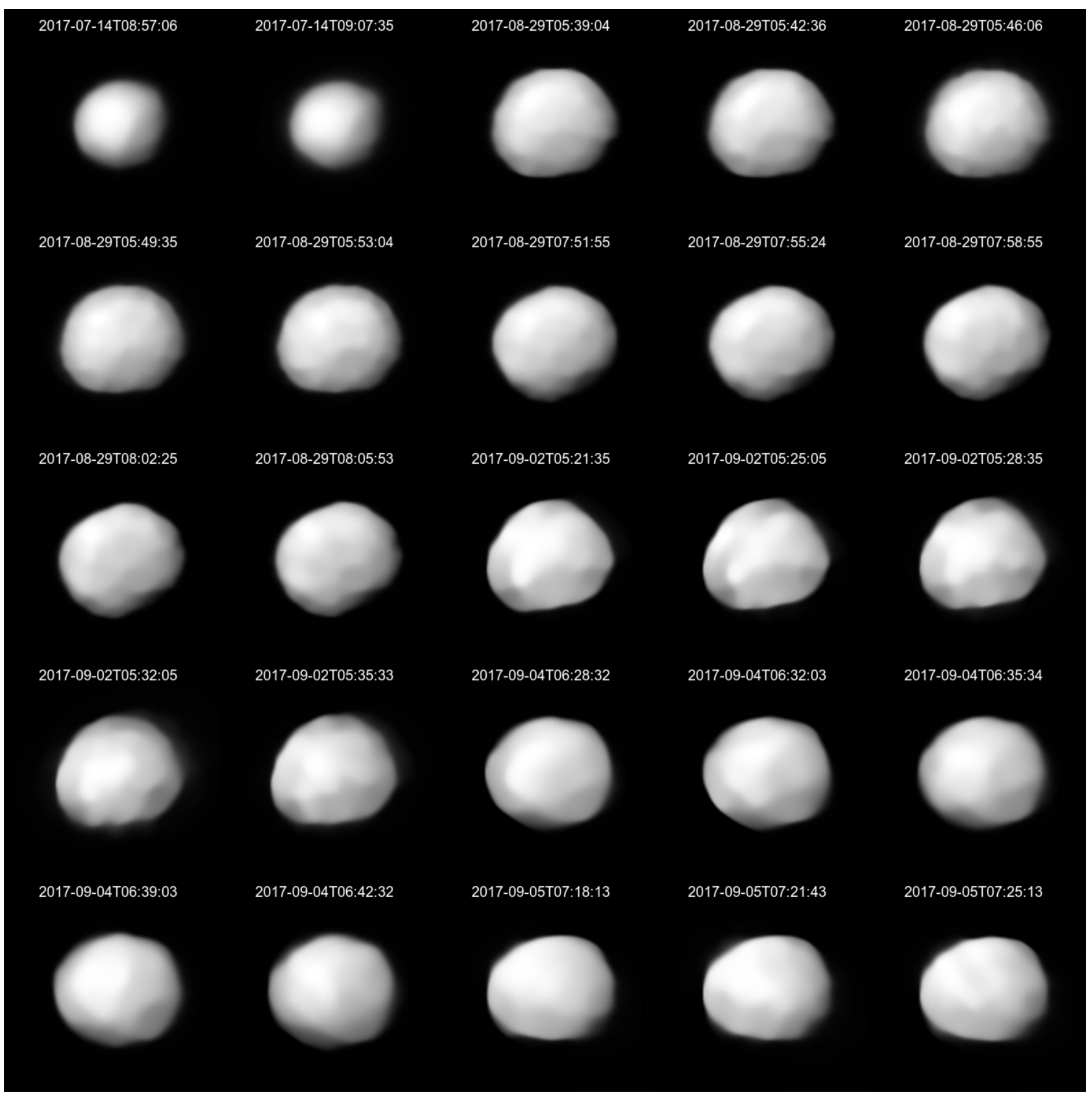

Fig. A.1. Full set of VLT/SPHERE/ZIMPOL images of (704) Interamnia obtained in August-September 2017. All images were deconvolved with the Mistral algorithm. Table A.1 contains full information about the data. 


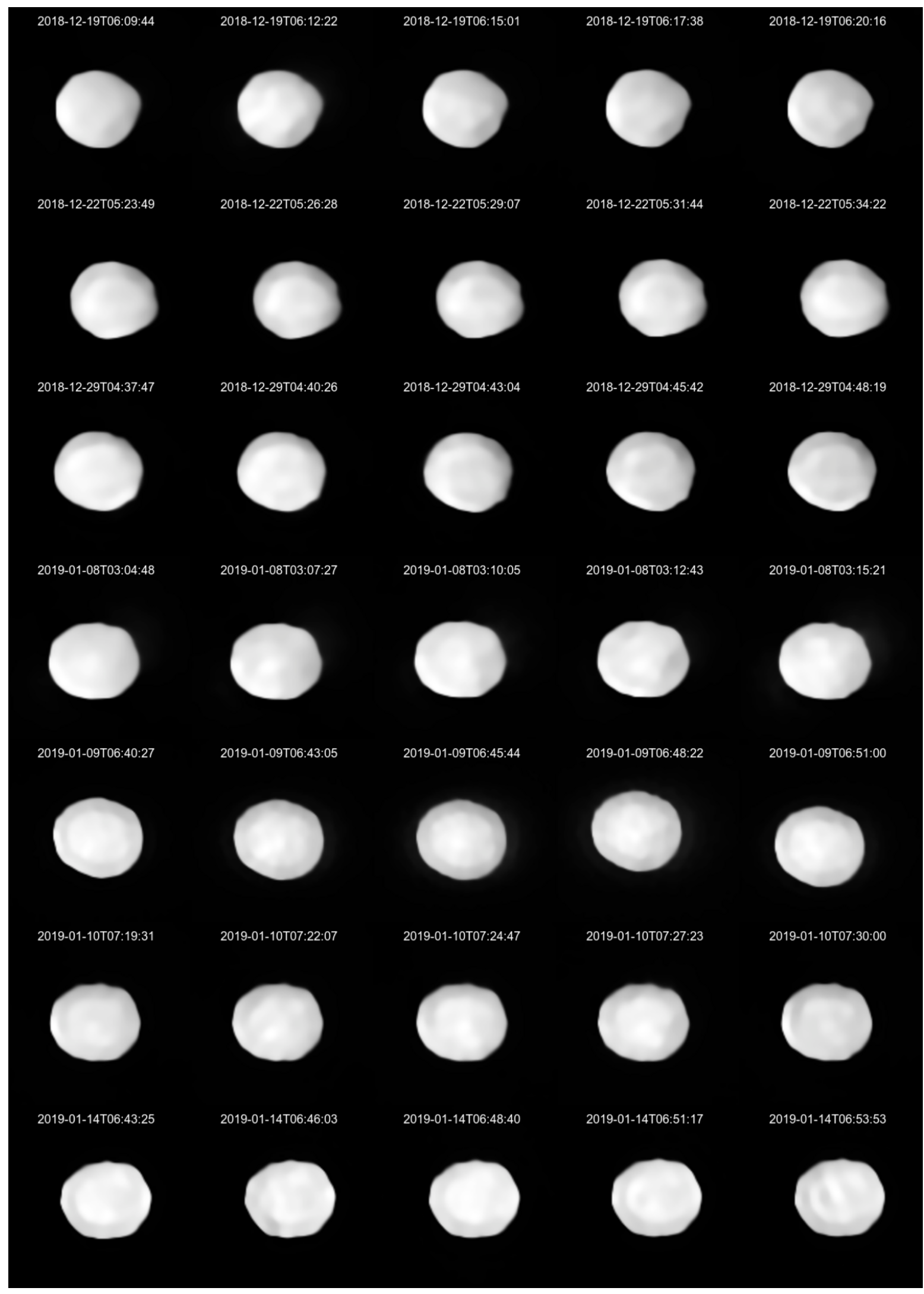

Fig. A.2. Full set of VLT/SPHERE/ZIMPOL images of (704) Interamnia obtained between December 2018 and January 2019. All images were deconvolved with the Mistral algorithm. Table A.1 contains full information about the data. 


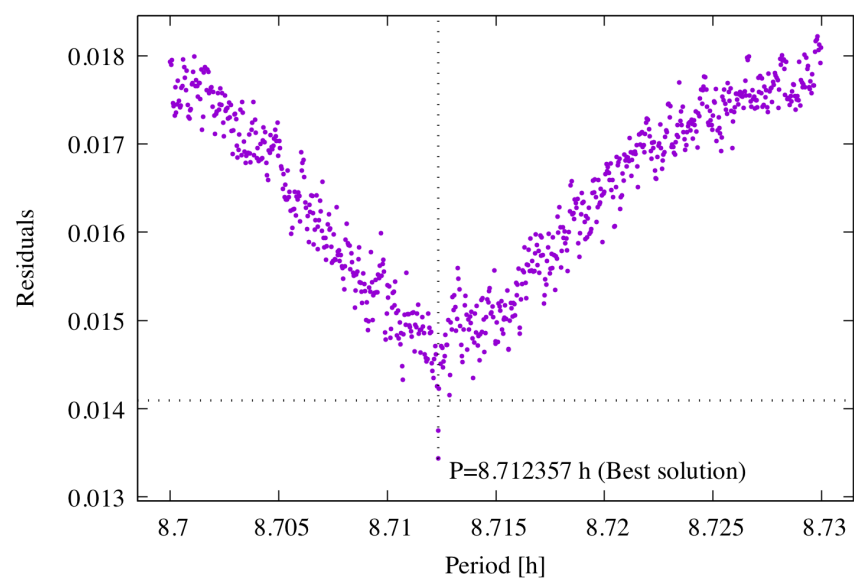

Fig. A.3. Periodogram for Interamnia: each point corresponds to a local minimum in the parameter space. The point with the lowest rms is the global minimum and the horizontal line indicates the rms threshold as defined in Hanuš et al. (2018).

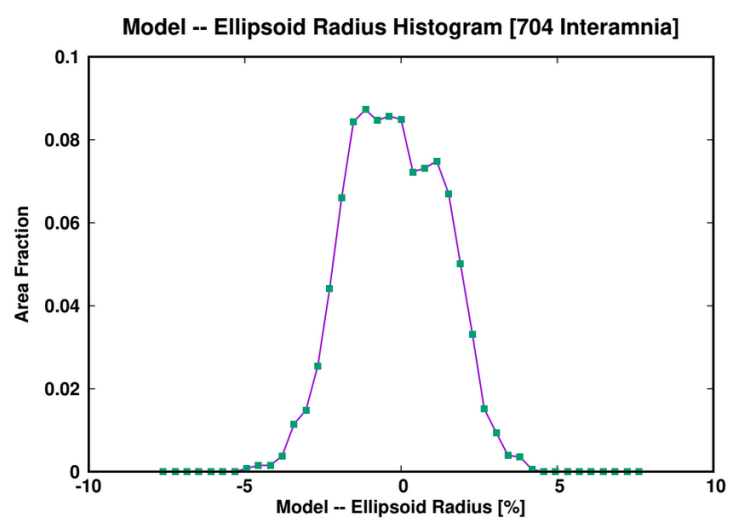

Fig. A.5. Distribution of residuals measured along the local normal direction between the ADAM shape model and the best-fitting ellipsoid.

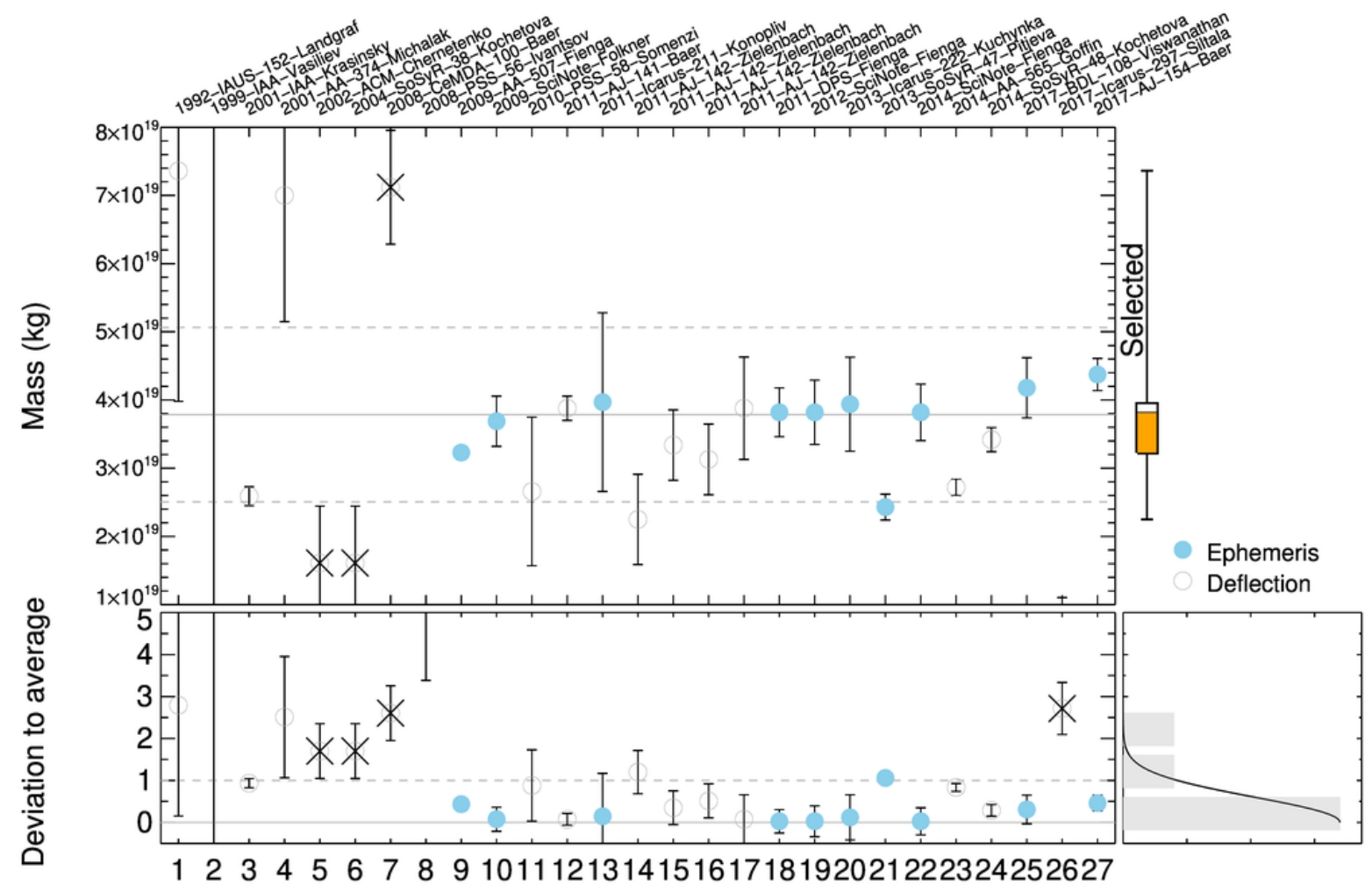

Fig. A.4. Mass estimates $(\mathcal{M})$ of (704) Interamnia collected in the literature. 
A\&A 633, A65 (2020)

Table A.1. VLT/SPHERE disk-resolved images obtained in the I filter by the ZIMPOL camera.

\begin{tabular}{|c|c|c|c|c|c|c|c|}
\hline Date & UT & $\begin{array}{c}\operatorname{Exp} \\
\text { (s) }\end{array}$ & Airmass & $\begin{array}{c}\Delta \\
(\mathrm{AU})\end{array}$ & $\begin{array}{c}r \\
(\mathrm{AU})\end{array}$ & $\begin{array}{c}\alpha \\
\left({ }^{\circ}\right)\end{array}$ & $\begin{array}{l}D_{\mathrm{a}} \\
\left({ }^{\prime \prime}\right)\end{array}$ \\
\hline 2017-07-14 & $8: 57: 06$ & 200 & 1.46 & 2.24 & 2.62 & 22.4 & 0.203 \\
\hline 2017-07-14 & $9: 07: 35$ & 200 & 1.45 & 2.24 & 2.62 & 22.4 & 0.203 \\
\hline 2017-08-29 & $5: 39: 04$ & 200 & 1.71 & 1.78 & 2.60 & 15.9 & 0.256 \\
\hline $2017-08-29$ & $5: 42: 36$ & 200 & 1.70 & 1.78 & 2.60 & 15.9 & 0.256 \\
\hline $2017-08-29$ & $5: 46: 06$ & 200 & 1.70 & 1.78 & 2.60 & 15.9 & 0.256 \\
\hline $2017-08-29$ & $5: 49: 35$ & 200 & 1.69 & 1.78 & 2.60 & 15.9 & 0.256 \\
\hline $2017-08-29$ & 5:53:04 & 200 & 1.69 & 1.78 & 2.60 & 15.9 & 0.256 \\
\hline $2017-08-29$ & $7: 51: 55$ & 200 & 1.88 & 1.78 & 2.60 & 15.9 & 0.256 \\
\hline $2017-08-29$ & $7: 55: 24$ & 200 & 1.89 & 1.78 & 2.60 & 15.9 & 0.256 \\
\hline 2017-08-29 & $7: 58: 55$ & 200 & 1.91 & 1.78 & 2.60 & 15.9 & 0.256 \\
\hline 2017-08-29 & $8: 02: 25$ & 200 & 1.93 & 1.78 & 2.60 & 15.9 & 0.256 \\
\hline 2017-08-29 & $8: 05: 53$ & 200 & 1.95 & 1.78 & 2.60 & 15.9 & 0.256 \\
\hline 2017-09-02 & $5: 21: 35$ & 200 & 1.72 & 1.76 & 2.60 & 15.0 & 0.259 \\
\hline 2017-09-02 & $5: 25: 05$ & 200 & 1.71 & 1.76 & 2.60 & 15.0 & 0.259 \\
\hline 2017-09-02 & $5: 28: 35$ & 200 & 1.71 & 1.76 & 2.60 & 15.0 & 0.259 \\
\hline 2017-09-02 & $5: 32: 05$ & 200 & 1.70 & 1.76 & 2.60 & 15.0 & 0.259 \\
\hline 2017-09-02 & $5: 35: 33$ & 200 & 1.70 & 1.76 & 2.60 & 15.0 & 0.259 \\
\hline 2017-09-04 & $6: 28: 32$ & 200 & 1.72 & 1.74 & 2.60 & 14.5 & 0.261 \\
\hline 2017-09-04 & $6: 32: 03$ & 200 & 1.73 & 1.74 & 2.60 & 14.5 & 0.261 \\
\hline 2017-09-04 & $6: 35: 34$ & 200 & 1.73 & 1.74 & 2.60 & 14.5 & 0.261 \\
\hline 2017-09-04 & $6: 39: 03$ & 200 & 1.74 & 1.74 & 2.60 & 14.5 & 0.261 \\
\hline 2017-09-04 & $6: 42: 32$ & 200 & 1.75 & 1.74 & 2.60 & 14.5 & 0.261 \\
\hline 2017-09-05 & $7: 18: 13$ & 200 & 1.89 & 1.74 & 2.60 & 14.3 & 0.261 \\
\hline 2017-09-05 & $7: 21: 43$ & 200 & 1.91 & 1.74 & 2.60 & 14.3 & 0.261 \\
\hline 2017-09-05 & $7: 25: 13$ & 200 & 1.92 & 1.74 & 2.60 & 14.3 & 0.261 \\
\hline 2018-12-19 & $6: 09: 44$ & 147 & 1.35 & 2.15 & 3.02 & 10.1 & 0.212 \\
\hline 2018-12-19 & $6: 12: 22$ & 147 & 1.35 & 2.15 & 3.02 & 10.1 & 0.212 \\
\hline 2018-12-19 & $6: 15: 01$ & 147 & 1.34 & 2.15 & 3.02 & 10.1 & 0.212 \\
\hline 2018-12-19 & $6: 17: 38$ & 147 & 1.34 & 2.15 & 3.02 & 10.1 & 0.212 \\
\hline 2018-12-19 & $6: 20: 16$ & 147 & 1.34 & 2.15 & 3.02 & 10.1 & 0.212 \\
\hline $2018-12-22$ & $5: 23: 49$ & 147 & 1.41 & 2.13 & 3.03 & 9.2 & 0.214 \\
\hline $2018-12-22$ & $5: 26: 28$ & 147 & 1.40 & 2.13 & 3.03 & 9.2 & 0.214 \\
\hline $2018-12-22$ & $5: 29: 07$ & 147 & 1.39 & 2.13 & 3.03 & 9.2 & 0.214 \\
\hline $2018-12-22$ & $5: 31: 44$ & 147 & 1.39 & 2.13 & 3.03 & 9.2 & 0.214 \\
\hline $2018-12-22$ & $5: 34: 22$ & 147 & 1.38 & 2.13 & 3.03 & 9.2 & 0.214 \\
\hline $2018-12-29$ & $4: 37: 47$ & 147 & 1.43 & 2.10 & 3.04 & 6.7 & 0.217 \\
\hline $2018-12-29$ & $4: 40: 26$ & 147 & 1.43 & 2.10 & 3.04 & 6.7 & 0.217 \\
\hline $2018-12-29$ & 4:43:04 & 147 & 1.42 & 2.10 & 3.04 & 6.7 & 0.217 \\
\hline $2018-12-29$ & $4: 45: 42$ & 147 & 1.41 & 2.10 & 3.04 & 6.7 & 0.217 \\
\hline $2018-12-29$ & $4: 48: 19$ & 147 & 1.40 & 2.10 & 3.04 & 6.7 & 0.217 \\
\hline 2019-01-08 & $3: 04: 48$ & 147 & 1.60 & 2.08 & 3.05 & 3.3 & 0.219 \\
\hline 2019-01-08 & $3: 07: 27$ & 147 & 1.59 & 2.08 & 3.05 & 3.3 & 0.219 \\
\hline 2019-01-08 & $3: 10: 05$ & 147 & 1.58 & 2.08 & 3.05 & 3.3 & 0.219 \\
\hline 2019-01-08 & $3: 12: 43$ & 147 & 1.56 & 2.08 & 3.05 & 3.3 & 0.219 \\
\hline 2019-01-08 & $3: 15: 21$ & 147 & 1.55 & 2.08 & 3.05 & 3.3 & 0.219 \\
\hline 2019-01-09 & $6: 40: 27$ & 147 & 1.42 & 2.08 & 3.05 & 2.9 & 0.219 \\
\hline 2019-01-09 & $6: 43: 05$ & 147 & 1.43 & 2.08 & 3.05 & 2.9 & 0.219 \\
\hline 2019-01-09 & $6: 45: 44$ & 147 & 1.44 & 2.08 & 3.05 & 2.9 & 0.219 \\
\hline 2019-01-09 & $6: 48: 22$ & 147 & 1.44 & 2.08 & 3.05 & 2.9 & 0.219 \\
\hline 2019-01-09 & $6: 51: 00$ & 147 & 1.45 & 2.08 & 3.05 & 2.9 & 0.219 \\
\hline 2019-01-10 & $7: 19: 31$ & 147 & 1.59 & 2.08 & 3.06 & 2.6 & 0.219 \\
\hline 2019-01-10 & $7: 22: 07$ & 147 & 1.61 & 2.08 & 3.06 & 2.6 & 0.219 \\
\hline 2019-01-10 & $7: 24: 47$ & 147 & 1.62 & 2.08 & 3.06 & 2.6 & 0.219 \\
\hline 2019-01-10 & $7: 27: 23$ & 147 & 1.64 & 2.08 & 3.06 & 2.6 & 0.219 \\
\hline 2019-01-10 & $7: 30: 00$ & 147 & 1.65 & 2.08 & 3.06 & 2.6 & 0.219 \\
\hline 2019-01-14 & $6: 43: 25$ & 147 & 1.51 & 2.08 & 3.06 & 2.0 & 0.219 \\
\hline 2019-01-14 & $6: 46: 03$ & 147 & 1.52 & 2.08 & 3.06 & 2.0 & 0.219 \\
\hline 2019-01-14 & $6: 48: 40$ & 147 & 1.53 & 2.08 & 3.06 & 2.0 & 0.219 \\
\hline 2019-01-14 & $6: 51: 17$ & 147 & 1.55 & 2.08 & 3.06 & 2.0 & 0.219 \\
\hline 2019-01-14 & $6: 53: 53$ & 147 & 1.56 & 2.08 & 3.06 & 2.0 & 0.219 \\
\hline
\end{tabular}

Notes. For each observation, the table gives the epoch, the exposure time, the airmass, the distance to the Earth $\Delta$ and the Sun $r$, the phase angle $\alpha$, and the angular diameter $D_{\mathrm{a}}$. 
Table A.2. Stellar occultations used for shape modeling, with individual observers names.

\begin{tabular}{l}
\hline \hline \multicolumn{1}{c}{ (704) Interamnia } \\
\hline 1996-12-17 \\
Bob Fried, Braeside Obs., Flagstaff, AZ, USA \\
Gary Goodman, Camarillo, CA, USA \\
Etscorn Obs., Socorro, NM, USA \\
Orange County A.S. Obs., Anza, CA, USA \\
F. Wright/Fulton Jr., Prescott, AZ, USA \\
Pierre Schwaar, Phoenix, AZ, USA \\
P. Maley/L Paller, Phoenix, AZ, USA \\
Sam Herchak, Mesa, AZ, USA \\
Table Mtn. Obs., Wrightwood, CA, USA \\
Ken Ziegler, Gila Obs., Globe, AZ, USA \\
Bill Peters, AZ, USA \\
\hline
\end{tabular}

$$
\text { 2003-3-23 }
$$

Yoshida Hidetoshi, Abashiri, Hokkaido, JP Kouda Masaki, Kamikita, Aomori, JP Imatani \& Takashi, Kitaura, Ibaraka, JP Sugawara Hitoshi, Ichinoseki, Iwate, JP Satou Toshirou, Ichinoseki, Iwate, JP Yokokawa Mikio, Motoyoshi, Miyagi, JP Konno Eitoshi, Hanaizumi, Iwate JP Sasaki Kazuo, Furukawa, Miyagi, JP Tonomura Yasuhiro, Tomiya, Miyagi, JP Okamoto Michiko, Rifu, Miyagi, JP Sakaki Chiyoaki, Sendai, Miyagi, JP Nagai Hideo, Sendai, Miyagi, JP Itou Yoshiharu, Aoba, Sendai, JP Ikeshita Ryo et al., Kawauchi, Sendai, JP Koishikawa Masahiro, Sendai, Miyagi, JP Watanabe, Akira, Sendai, Miyagi, JP Miyamoto Atsushi et al, Adachi, JP Sugai Hideo, Zao-hango, Yamagata, JP Fujita Mitsuhiro, Shiroishi, Miyagi, JP Nihei Hajime, Nanyo, Yamagata, JP Tanaka Takashi, Zushi, Kanagawa, JP Sato Tsutomu, Marumori, Miyagi, JP Ootsuki Isao, Marumori, Miyagi, JP David Tholen, Turtle Bay, Oahu, Hawaii, USA Sato Hikaru, Fukushima, JP Sato Makoto, Haranomachi, Fukushima, JP Rebecca Sydney, Honolua Bay, Maui, HI Bedient et al., Foster Village, Hawaii, USA Hamanowa et al., Koriyama City, JP Usuki Ken-ichi, Niitsuru, Fukushima, JP Lewis Roberts, Haleakala, Hawaii, USA Watanabe et al., Takine, Fukushima, JP Sato Hirohisa, Sukagawa, Fukushima, JP David Dunham, Makena, Maui, HI, USA B. Brevoort, Hawaii, USA

Tsuchikawa Akira, Yanagida, JP

S. Bus, Mauna Kea, Hawaii, USA

R. Savalle, Mauna Kea, Hawaii, USA

Tomioka Hiroyuki, Hitachi, Ibaraki, JP P. Maley, Hawaii, USA Yaeza Akira,Moriyama-cho, Hitachi, JP

E. Cleintuar, Hawaii, USA

S. O’Meara, Mauna Loa, USA

W. Fukunaga, Hawaii, USA
Table A.2. continued.

\begin{tabular}{l}
\hline \hline V. Fukunaga, Hawaii, USA \\
J. Swatek, Hawaii, USA \\
Uehara Sadaharu, Ibaraki, JP \\
\hline Observer \\
\hline Kuboniwa Atasushi, Ushika,Ibaraka, JP \\
Kita Nobusuke, Kashiwa, Chiba, JP \\
Takashima et al, Kashiwa, Chiba, JP \\
Momose Masahiko, Shiojiri, Nagano, JP \\
Kaneko Sakae, Sakura, Chiba, JP \\
Nakanishi Akio, Itabashi, Tokyo, JP \\
Ishida Masayuki, Kanazu, Fukui, JP \\
Kitazaki Katsuhiko, Tokyo, JP \\
Ida Miyoshi, Muraoka, Fukui, JP \\
Suzuki Satoshi, Yokohama,Kanagawa, JP \\
Hirose Yoji, Chigasaki, Kanagawa, JP \\
Sugiyama Yukihiro, Hiratsuka, JP \\
Yoneyama Seiichi, Ogaki, Gifu, JP \\
Oribe Takaaki et al., Saji, Tottori, JP \\
\hline \\
R. Cadmus, Grinnell, IA, USA \\
J. Centala, Marion, IA, USA \\
W. Osborn/C. Tycner, Mt. Pleasant, MI, USA \\
P. Maley, Bernalillo, NM, USA \\
K. McKeown, Los Lunas, NM, USA \\
D. Dunham, Dubuque IA, USA \\
\hline \\
N. Smith, Trenton, GA, USA \\
T. Blank/M. Pacht, Taftsville, VT, USA \\
S. Conard, Gamber, MD, USA \\
A. Scheck, Scaggsville, MD, USA \\
C. Ellington, Owings, MD, USA \\
\hline
\end{tabular}


Table A.3. Optical disk-integrated lightcurves used for ADAM shape modeling.

\begin{tabular}{|c|c|c|c|c|c|c|c|}
\hline $\mathrm{N}$ & Epoch & $N_{\mathrm{p}}$ & $\begin{array}{c}\Delta \\
(\mathrm{AU})\end{array}$ & $\begin{array}{c}r \\
(\mathrm{AU})\end{array}$ & $\begin{array}{l}\varphi \\
\left({ }^{\circ}\right)\end{array}$ & Filter & Reference \\
\hline 1 & $1964-11-22.7$ & 81 & 1.82 & 2.75 & 8.1 & $V$ & Yang et al. (1965) \\
\hline 2 & $1964-11-23.7$ & 57 & 1.81 & 2.75 & 7.7 & $V$ & Yang et al. (1965) \\
\hline 3 & $1969-08-16.5$ & 8 & 2.18 & 2.58 & 22.6 & $V$ & Tempesti (1975) \\
\hline 4 & 1969-08-20.6 & 7 & 2.14 & 2.58 & 22.3 & $V$ & Tempesti (1975) \\
\hline 5 & $1969-08-21.5$ & 12 & 2.13 & 2.58 & 22.2 & $V$ & Tempesti (1975) \\
\hline 6 & 1969-09-08.5 & 12 & 1.95 & 2.59 & 19.9 & $V$ & Tempesti (1975) \\
\hline 7 & $1969-10-05.6$ & 13 & 1.74 & 2.60 & 13.9 & $V$ & Tempesti (1975) \\
\hline 8 & $1969-10-15.4$ & 10 & 1.70 & 2.60 & 11.4 & $V$ & Tempesti (1975) \\
\hline 9 & 1969-11-01.4 & 11 & 1.68 & 2.61 & 9.1 & $V$ & Tempesti (1975) \\
\hline 10 & $1969-11-03.4$ & 15 & 1.68 & 2.61 & 9.2 & $V$ & Tempesti (1975) \\
\hline 11 & $1969-11-10.5$ & 23 & 1.69 & 2.62 & 9.8 & $V$ & Tempesti (1975) \\
\hline 12 & $1974-08-27.1$ & 29 & 1.71 & 2.62 & 12.2 & $V$ & Lustig \& Hahn (1976) \\
\hline 13 & $1974-08-28.0$ & 91 & 1.71 & 2.62 & 12.1 & $V$ & Lustig \& Hahn (1976) \\
\hline 14 & $1974-08-29.0$ & 92 & 1.71 & 2.62 & 11.9 & $V$ & Lustig \& Hahn (1976) \\
\hline 15 & 1990-08-01.9 & 76 & 1.86 & 2.68 & 15.5 & $V$ & Shevchenko et al. (1992) \\
\hline 16 & $1993-03-21.6$ & 77 & 2.55 & 3.47 & 7.6 & $V$ & Michalowski et al. (1995) \\
\hline 17 & $1993-03-23.6$ & 73 & 2.56 & 3.47 & 7.9 & $V$ & Michalowski et al. (1995) \\
\hline 18 & $1996-12-13.2$ & 512 & 1.83 & 2.74 & 9.6 & $V$ & Satō et al. (2014) \\
\hline 19 & $1996-12-13.3$ & 354 & 1.83 & 2.74 & 9.6 & $R$ & Satō et al. (2014) \\
\hline 20 & $2003-03-31.9$ & 29 & 2.85 & 3.20 & 17.8 & $C$ & Stephane Charbonnel \\
\hline 21 & 2003-03-31.9 & 55 & 2.85 & 3.20 & 17.8 & $C$ & Nathanal Berger \\
\hline 22 & $2006-06-04.1$ & 30 & 2.46 & 2.79 & 21.0 & $C$ & Arnaud Leroy \\
\hline 23 & 2006-07-21 & 59 & 1.89 & 2.74 & 14.3 & $C$ & Grice et al. (2017) \\
\hline 24 & $2006-07-25$ & 53 & 1.86 & 2.73 & 13.3 & $C$ & Grice et al. (2017) \\
\hline 25 & $2006-07-25$ & 54 & 1.86 & 2.73 & 13.3 & $C$ & Grice et al. (2017) \\
\hline 26 & $2006-07-26$ & 38 & 1.85 & 2.73 & 13.1 & $C$ & Grice et al. (2017) \\
\hline 27 & $2006-07-26$ & 40 & 1.85 & 2.73 & 13.1 & $C$ & Grice et al. (2017) \\
\hline 28 & $2006-07-27$ & 50 & 1.85 & 2.73 & 12.8 & $C$ & Grice et al. (2017) \\
\hline 29 & 2006-07-27 & 55 & 1.85 & 2.73 & 12.8 & $C$ & Grice et al. (2017) \\
\hline 30 & $2006-07-28$ & 56 & 1.84 & 2.73 & 12.6 & $C$ & Grice et al. (2017) \\
\hline 31 & $2006-07-28$ & 56 & 1.84 & 2.73 & 12.6 & $C$ & Grice et al. (2017) \\
\hline 32 & 2006-07-29 & 54 & 1.83 & 2.73 & 12.3 & $C$ & Grice et al. (2017) \\
\hline 33 & 2006-07-29 & 58 & 1.83 & 2.73 & 12.3 & $C$ & Grice et al. (2017) \\
\hline 34 & $2006-07-30$ & 59 & 1.82 & 2.72 & 12.1 & $C$ & Grice et al. (2017) \\
\hline 35 & $2006-07-30$ & 59 & 1.82 & 2.72 & 12.1 & $C$ & Grice et al. (2017) \\
\hline 36 & 2006-07-31 & 46 & 1.82 & 2.72 & 11.8 & $C$ & Grice et al. (2017) \\
\hline 37 & 2006-07-31 & 59 & 1.82 & 2.72 & 11.8 & $C$ & Grice et al. (2017) \\
\hline 38 & 2006-08-02 & 64 & 1.81 & 2.72 & 11.3 & $C$ & Grice et al. (2017) \\
\hline 39 & 2006-08-04 & 89 & 1.79 & 2.72 & 10.8 & $C$ & Grice et al. (2017) \\
\hline 40 & $2006-08-05$ & 51 & 1.79 & 2.72 & 10.6 & $C$ & Grice et al. (2017) \\
\hline 41 & $2006-08-05$ & 87 & 1.79 & 2.72 & 10.6 & $C$ & Grice et al. (2017) \\
\hline 42 & $2006-08-06$ & 61 & 1.78 & 2.72 & 10.3 & $C$ & Grice et al. (2017) \\
\hline 43 & 2006-08-06 & 89 & 1.78 & 2.72 & 10.3 & $C$ & Grice et al. (2017) \\
\hline 44 & 2006-08-07 & 62 & 1.78 & 2.72 & 10.1 & $C$ & Grice et al. (2017) \\
\hline 45 & 2006-08-07 & 88 & 1.78 & 2.72 & 10.1 & $C$ & Grice et al. (2017) \\
\hline 46 & 2006-08-12 & 105 & 1.76 & 2.71 & 9.0 & $C$ & Grice et al. (2017) \\
\hline 47 & 2006-08-12 & 118 & 1.76 & 2.71 & 9.0 & $C$ & Grice et al. (2017) \\
\hline 48 & $2006-08-13$ & 67 & 1.75 & 2.71 & 8.9 & $C$ & Grice et al. (2017) \\
\hline 49 & $2006-08-13$ & 91 & 1.75 & 2.71 & 8.9 & $C$ & Grice et al. (2017) \\
\hline 50 & 2006-08-19 & 49 & 1.74 & 2.70 & 8.1 & $C$ & Grice et al. (2017) \\
\hline 51 & $2006-08-20$ & 41 & 1.74 & 2.70 & 8.0 & $C$ & Grice et al. (2017) \\
\hline 52 & 2006-08-22 & 54 & 1.73 & 2.70 & 7.9 & $C$ & Grice et al. (2017) \\
\hline 53 & $2006-08-25$ & 42 & 1.73 & 2.70 & 8.0 & $C$ & Grice et al. (2017) \\
\hline 54 & $2006-08-30$ & 52 & 1.73 & 2.69 & 8.4 & $C$ & Grice et al. (2017) \\
\hline 55 & $2006-08-31$ & 67 & 1.73 & 2.69 & 8.5 & $C$ & Grice et al. (2017) \\
\hline
\end{tabular}

Notes. For each lightcurve, the table gives the epoch, the number of individual measurements $N_{\mathrm{p}}$, asteroid's distances to the Earth $\Delta$ and the Sun $r$, phase angle $\varphi$, photometric filter and observation information. Gaia-GOSA (Gaia-Ground-based Observational Service for Asteroids, www. gaiagosa.eu). 
Table A.3. continued.

\begin{tabular}{|c|c|c|c|c|c|c|c|}
\hline $\mathrm{N}$ & Epoch & $N_{\mathrm{p}}$ & $\begin{array}{c}\Delta \\
(\mathrm{AU})\end{array}$ & $\begin{array}{c}r \\
(\mathrm{AU})\end{array}$ & $\begin{array}{l}\varphi \\
\left(^{\circ}\right)\end{array}$ & Filter & Reference \\
\hline 56 & $2006-08-32.0$ & 236 & 1.73 & 2.69 & 8.5 & $C$ & Dominique Suys, Hugo Riemis, Jan Vantomme \\
\hline 57 & 2006-09-02 & 52 & 1.74 & 2.69 & 8.9 & $C$ & Grice et al. (2017) \\
\hline 58 & $2006-09-12$ & 57 & 1.77 & 2.68 & 11.1 & $C$ & Grice et al. (2017) \\
\hline 59 & 2006-09-14 & 50 & 1.77 & 2.68 & 11.6 & $C$ & Grice et al. (2017) \\
\hline 60 & 2006-09-16 & 46 & 1.78 & 2.68 & 12.1 & $C$ & Grice et al. (2017) \\
\hline 61 & 2006-09-19 & 38 & 1.80 & 2.67 & 12.9 & $C$ & Grice et al. (2017) \\
\hline 62 & $2006-09-22$ & 46 & 1.82 & 2.67 & 13.7 & $C$ & Grice et al. (2017) \\
\hline 63 & 2006-09-24 & 47 & 1.83 & 2.67 & 14.3 & $C$ & Grice et al. (2017) \\
\hline 64 & 2006-09-26 & 47 & 1.84 & 2.67 & 14.8 & $C$ & Grice et al. (2017) \\
\hline 65 & $2006-09-27$ & 38 & 1.85 & 2.67 & 15.0 & $C$ & Grice et al. (2017) \\
\hline 66 & 2006-10-08 & 43 & 1.94 & 2.66 & 17.6 & $C$ & Grice et al. (2017) \\
\hline 67 & 2007-10-18 & 39 & 2.29 & 2.78 & 19.8 & $C$ & Grice et al. (2017) \\
\hline 68 & 2007-11-22 & 80 & 1.96 & 2.82 & 11.7 & $C$ & Grice et al. (2017) \\
\hline 69 & $2007-12-27.9$ & 34 & 1.90 & 2.87 & 3.1 & $C$ & Jean-Francois Coliac \\
\hline 70 & $2008-12-28$ & 58 & 2.94 & 3.37 & 16.2 & $C$ & Grice et al. (2017) \\
\hline 71 & 2009-01-03 & 59 & 2.87 & 3.37 & 15.5 & $C$ & Grice et al. (2017) \\
\hline 72 & 2009-01-04 & 58 & 2.85 & 3.38 & 15.4 & $C$ & Grice et al. (2017) \\
\hline 73 & 2009-01-17 & 61 & 2.71 & 3.39 & 13.5 & $C$ & Grice et al. (2017) \\
\hline 74 & 2009-01-18 & 55 & 2.70 & 3.39 & 13.4 & $C$ & Grice et al. (2017) \\
\hline 75 & $2009-01-20$ & 53 & 2.68 & 3.39 & 13.0 & $C$ & Grice et al. (2017) \\
\hline 76 & $2009-01-20$ & 73 & 2.68 & 3.39 & 13.0 & $C$ & Grice et al. (2017) \\
\hline 77 & 2009-01-21 & 58 & 2.67 & 3.39 & 12.8 & $C$ & Grice et al. (2017) \\
\hline 78 & $2009-01-23$ & 51 & 2.65 & 3.39 & 12.4 & $C$ & Grice et al. (2017) \\
\hline 79 & $2009-01-24$ & 55 & 2.65 & 3.39 & 12.2 & $C$ & Grice et al. (2017) \\
\hline 80 & $2009-01-24$ & 77 & 2.64 & 3.39 & 12.2 & $C$ & Grice et al. (2017) \\
\hline 81 & $2009-01-25$ & 56 & 2.64 & 3.40 & 12.0 & $C$ & Grice et al. (2017) \\
\hline 82 & $2009-01-26$ & 61 & 2.63 & 3.40 & 11.8 & $C$ & Grice et al. (2017) \\
\hline 83 & $2009-01-27$ & 71 & 2.62 & 3.40 & 11.6 & $C$ & Grice et al. (2017) \\
\hline 84 & $2009-01-28$ & 73 & 2.61 & 3.40 & 11.4 & $C$ & Grice et al. (2017) \\
\hline 85 & $2009-01-31$ & 60 & 2.59 & 3.40 & 10.8 & $C$ & Grice et al. (2017) \\
\hline 86 & $2009-02-01$ & 37 & 2.58 & 3.40 & 10.6 & $C$ & Grice et al. (2017) \\
\hline 87 & $2009-02-02$ & 77 & 2.58 & 3.40 & 10.4 & $C$ & Grice et al. (2017) \\
\hline 88 & $2009-02-16$ & 46 & 2.50 & 3.42 & 7.5 & $C$ & Grice et al. (2017) \\
\hline 89 & 2009-02-18 & 85 & 2.50 & 3.42 & 7.1 & $C$ & Grice et al. (2017) \\
\hline 90 & 2009-02-19 & 46 & 2.49 & 3.42 & 7.0 & $C$ & Grice et al. (2017) \\
\hline 91 & $2009-02-21$ & 81 & 2.49 & 3.42 & 6.7 & $C$ & Grice et al. (2017) \\
\hline 92 & $2009-02-21$ & 84 & 2.49 & 3.42 & 6.7 & $C$ & Grice et al. (2017) \\
\hline 93 & $2009-02-22$ & 47 & 2.49 & 3.42 & 6.6 & $C$ & Grice et al. (2017) \\
\hline 94 & $2009-02-22$ & 63 & 2.49 & 3.42 & 6.6 & $C$ & Grice et al. (2017) \\
\hline 95 & $2009-02-26$ & 40 & 2.48 & 3.42 & 6.2 & $C$ & Grice et al. (2017) \\
\hline 96 & $2009-02-27$ & 48 & 2.48 & 3.42 & 6.1 & $C$ & Grice et al. (2017) \\
\hline 97 & 2009-03-01 & 50 & 2.48 & 3.43 & 6.1 & $C$ & Grice et al. (2017) \\
\hline 98 & 2009-03-02 & 83 & 2.48 & 3.43 & 6.1 & $C$ & Grice et al. (2017) \\
\hline 99 & 2009-03-04 & 55 & 2.49 & 3.43 & 6.1 & $C$ & Grice et al. (2017) \\
\hline 100 & 2009-03-13 & 93 & 2.51 & 3.44 & 7.0 & $C$ & Grice et al. (2017) \\
\hline 101 & 2009-03-14 & 65 & 2.51 & 3.44 & 7.2 & $C$ & Grice et al. (2017) \\
\hline 102 & $2009-03-16$ & 53 & 2.52 & 3.44 & 7.6 & $C$ & Grice et al. (2017) \\
\hline 103 & $2009-03-17$ & 61 & 2.53 & 3.44 & 7.7 & $C$ & Grice et al. (2017) \\
\hline 104 & 2009-03-19 & 64 & 2.54 & 3.44 & 8.1 & $C$ & Grice et al. (2017) \\
\hline 105 & $2009-03-20$ & 47 & 2.54 & 3.44 & 8.3 & $C$ & Grice et al. (2017) \\
\hline 106 & $2009-03-20$ & 79 & 2.54 & 3.44 & 8.3 & $C$ & Grice et al. (2017) \\
\hline 107 & 2009-03-21 & 42 & 2.55 & 3.44 & 8.5 & $C$ & Grice et al. (2017) \\
\hline 108 & $2009-03-21$ & 71 & 2.55 & 3.44 & 8.5 & $C$ & Grice et al. (2017) \\
\hline 109 & $2009-03-22$ & 49 & 2.55 & 3.44 & 8.8 & $C$ & Grice et al. (2017) \\
\hline 110 & $2009-03-22$ & 76 & 2.55 & 3.44 & 8.7 & $C$ & Grice et al. (2017) \\
\hline 111 & $2009-03-23$ & 65 & 2.56 & 3.44 & 8.9 & $C$ & Grice et al. (2017) \\
\hline 112 & $2009-03-28$ & 85 & 2.59 & 3.45 & 10.0 & $C$ & Grice et al. (2017) \\
\hline 113 & 2009-03-29 & 83 & 2.60 & 3.45 & 10.2 & $C$ & Grice et al. (2017) \\
\hline 114 & $2009-03-30$ & 36 & 2.61 & 3.45 & 10.4 & $C$ & Grice et al. (2017) \\
\hline
\end{tabular}


Table A.3. continued.

\begin{tabular}{|c|c|c|c|c|c|c|c|}
\hline $\mathrm{N}$ & Epoch & $N_{\mathrm{p}}$ & $\begin{array}{c}\Delta \\
(\mathrm{AU})\end{array}$ & $\begin{array}{c}r \\
(\mathrm{AU})\end{array}$ & $\begin{array}{l}\varphi \\
\left(^{\circ}\right)\end{array}$ & Filter & Reference \\
\hline 115 & 2009-03-30 & 74 & 2.61 & 3.45 & 10.4 & $C$ & Grice et al. (2017) \\
\hline 116 & 2009-03-30 & 77 & 2.61 & 3.45 & 10.4 & $C$ & Grice et al. (2017) \\
\hline 117 & 2009-03-31 & 52 & 2.62 & 3.45 & 10.6 & $C$ & Grice et al. (2017) \\
\hline 118 & 2009-04-01 & 75 & 2.63 & 3.45 & 10.8 & $C$ & Grice et al. (2017) \\
\hline 119 & 2009-04-01 & 77 & 2.63 & 3.45 & 10.8 & $C$ & Grice et al. (2017) \\
\hline 120 & 2009-04-02 & 75 & 2.64 & 3.45 & 11.0 & $C$ & Grice et al. (2017) \\
\hline 121 & 2009-04-12 & 71 & 2.73 & 3.46 & 12.9 & $C$ & Grice et al. (2017) \\
\hline 122 & 2009-05-12 & 35 & 3.11 & 3.48 & 16.5 & $C$ & Grice et al. (2017) \\
\hline 123 & 2009-05-12 & 35 & 3.11 & 3.48 & 16.5 & $C$ & Grice et al. (2017) \\
\hline 124 & 2011-06-02 & 51 & 2.23 & 2.98 & 15.2 & $C$ & Grice et al. (2017) \\
\hline 125 & 2011-06-12 & 50 & 2.12 & 2.97 & 12.8 & $C$ & Grice et al. (2017) \\
\hline 126 & 2011-06-12 & 62 & 2.12 & 2.97 & 12.8 & $C$ & Grice et al. (2017) \\
\hline 127 & 2011-06-13 & 57 & 2.11 & 2.97 & 12.5 & $C$ & Grice et al. (2017) \\
\hline 128 & 2011-06-13 & 74 & 2.11 & 2.97 & 12.5 & $C$ & Grice et al. (2017) \\
\hline 129 & 2011-06-20 & 41 & 2.04 & 2.96 & 10.4 & $C$ & Grice et al. (2017) \\
\hline 130 & 2011-06-21 & 40 & 2.03 & 2.95 & 10.1 & $C$ & Grice et al. (2017) \\
\hline 131 & 2011-07-08 & 39 & 1.93 & 2.93 & 4.5 & $C$ & Grice et al. (2017) \\
\hline 132 & 2011-07-09 & 47 & 1.93 & 2.93 & 4.1 & $C$ & Grice et al. (2017) \\
\hline 133 & 2011-07-09 & 69 & 1.93 & 2.93 & 4.2 & $C$ & Grice et al. (2017) \\
\hline 134 & $2011-07-10$ & 41 & 1.92 & 2.93 & 3.8 & $C$ & Grice et al. (2017) \\
\hline 135 & $2011-07-10$ & 79 & 1.92 & 2.93 & 3.8 & $C$ & Grice et al. (2017) \\
\hline 136 & 2011-07-18 & 41 & 1.90 & 2.91 & 2.5 & $C$ & Grice et al. (2017) \\
\hline 137 & 2011-07-31 & 39 & 1.91 & 2.89 & 6.0 & $C$ & Grice et al. (2017) \\
\hline 138 & 2011-08-01 & 39 & 1.91 & 2.89 & 6.4 & $C$ & Grice et al. (2017) \\
\hline 139 & 2011-08-06 & 45 & 1.93 & 2.89 & 8.1 & $C$ & Grice et al. (2017) \\
\hline 140 & 2012-09-17.0 & 151 & 2.05 & 2.62 & 20.6 & $C$ & K. Sobkowiak, Borowiec, Poland \\
\hline 141 & 2012-09-18.9 & 17 & 2.03 & 2.62 & 20.3 & $C$ & J. Nadolny, Borowiec, Poland \\
\hline 142 & 2012-09-24.0 & 10 & 1.98 & 2.62 & 19.4 & $C$ & J. Nadolny, Borowiec, Poland \\
\hline 143 & $2012-10-01.0$ & 83 & 1.91 & 2.63 & 18.1 & $C$ & M. Bronikowska, Borowiec, Poland \\
\hline 144 & $2012-11-29.9$ & 45 & 1.75 & 2.67 & 9.3 & $C$ & Francisco Soldan \\
\hline 145 & 2012-12-01.9 & 48 & 1.76 & 2.67 & 9.8 & $C$ & Francisco Soldan \\
\hline 146 & 2012-12-02.9 & 59 & 1.77 & 2.68 & 10.1 & $C$ & Francisco Soldan \\
\hline 147 & 2012-12-08.9 & 29 & 1.81 & 2.68 & 11.7 & $C$ & Francisco Soldan \\
\hline 148 & $2012-12-26.0$ & 18 & 1.96 & 2.70 & 16.1 & $C$ & Francisco Soldan \\
\hline 149 & $2012-12-26.0$ & 24 & 1.96 & 2.70 & 16.1 & $C$ & Francisco Soldan \\
\hline 150 & $2012-12-26.8$ & 174 & 1.97 & 2.70 & 16.3 & $C$ & Francisco Soldan \\
\hline 151 & $2012-12-27.8$ & 145 & 1.98 & 2.70 & 16.6 & $C$ & Francisco Soldan \\
\hline 152 & $2012-12-28.8$ & 23 & 1.99 & 2.70 & 16.8 & $C$ & Francisco Soldan \\
\hline 153 & $2012-12-28.8$ & 55 & 1.99 & 2.70 & 16.8 & $C$ & Francisco Soldan \\
\hline 154 & 2016-05-23.1 & 52 & 2.27 & 3.18 & 9.3 & $C$ & Raul Melia, Cordoba, Argentina \\
\hline 155 & 2016-06-15.1 & 52 & 2.14 & 3.15 & 2.3 & $C$ & Carlos Colazo, Cordoba, Argentina \\
\hline 156 & 2016-06-16.2 & 42 & 2.14 & 3.15 & 2.1 & $C$ & Carlos Colazo, Cordoba, Argentina \\
\hline 157 & 2016-07-25.9 & 51 & 2.26 & 3.09 & 12.9 & $C$ & A. Marciniak, Obs. del Teide, Spain \\
\hline 158 & 2017-07-16.4 & 149 & 2.22 & 2.62 & 22.3 & $V$ & Warner (2018) \\
\hline 159 & 2017-07-17.4 & 172 & 2.21 & 2.62 & 22.2 & $V$ & Warner (2018) \\
\hline 160 & $2017-07-18.4$ & 156 & 2.20 & 2.62 & 22.2 & $V$ & Warner (2018) \\
\hline 161 & 2017-07-21.4 & 201 & 2.16 & 2.62 & 22.0 & $V$ & Warner (2018) \\
\hline 162 & 2017-07-22.4 & 196 & 2.15 & 2.62 & 21.9 & $V$ & Warner (2018) \\
\hline 163 & 2017-07-23.0 & 29 & 2.14 & 2.62 & 21.8 & $r$ & R. Szakats, Piszkes Obs., Hungary \\
\hline 164 & 2017-08-01.0 & 47 & 2.04 & 2.61 & 20.9 & $C$ & Adrian Jones, Gaia-GOSA \\
\hline 165 & $2017-08-12.0$ & 23 & 1.93 & 2.61 & 19.3 & $R$ & R. Szakats, Piszkes Obs., Hungary \\
\hline 166 & $2017-08-14.0$ & 35 & 1.91 & 2.61 & 19.0 & $R$ & R. Szakats, Piszkes Obs., Hungary \\
\hline 167 & $2017-08-15.1$ & 17 & 1.90 & 2.60 & 18.8 & $R$ & R. Szakats, Piszkes Obs., Hungary \\
\hline 168 & $2017-08-16.0$ & 66 & 1.89 & 2.60 & 18.6 & $R$ & R. Szakats, Piszkes Obs., Hungary \\
\hline 169 & 2017-10-11.9 & 38 & 1.67 & 2.59 & 11.1 & $r$ & R. Szakats, Piszkes Obs., Hungary \\
\hline 170 & $2017-10-16.8$ & 139 & 1.69 & 2.59 & 11.8 & $R$ & R. Duffard, La Sagra, Spain \\
\hline 171 & 2017-11-08.9 & 154 & 1.83 & 2.59 & 16.8 & $R$ & R. Duffard, La Sagra, Spain \\
\hline 172 & $2017-11-15.9$ & 276 & 1.89 & 2.59 & 18.2 & $R$ & R. Duffard, La Sagra, Spain \\
\hline 173 & 2017-11-16.9 & 188 & 1.90 & 2.59 & 18.4 & $R$ & R. Duffard, La Sagra, Spain \\
\hline
\end{tabular}


Table A.3. continued.

\begin{tabular}{llrrrrll}
\hline \hline $\mathrm{N}$ & \multicolumn{1}{c}{ Epoch } & $N_{\mathrm{p}}$ & $\begin{array}{c}\Delta \\
(\mathrm{AU})\end{array}$ & $\begin{array}{c}r \\
(\mathrm{AU})\end{array}$ & $\begin{array}{c}\varphi \\
\left(^{\circ}\right)\end{array}$ & Filter & Reference \\
\hline 174 & $2017-12-4.0$ & 76 & 2.07 & 2.59 & 20.9 & $C$ & D. Molina, Gaia-GOSA \\
175 & $2017-7-14.1$ & 58 & 2.25 & 2.62 & 22.4 & $C$ & D. Molina, Gaia-GOSA \\
176 & $2017-7-16.2$ & 63 & 2.22 & 2.62 & 22.3 & $C$ & D. Molina, Gaia-GOSA \\
177 & $2017-7-23.2$ & 48 & 2.14 & 2.62 & 21.8 & $C$ & D. Molina, Gaia-GOSA \\
178 & $2017-7-26.2$ & 35 & 2.11 & 2.62 & 21.6 & $C$ & D. Molina, Gaia-GOSA \\
179 & $2017-7-27.2$ & 58 & 2.10 & 2.62 & 21.5 & $C$ & D. Molina, Gaia-GOSA \\
180 & $2017-8-6.1$ & 187 & 1.99 & 2.61 & 20.3 & $C$ & A. Jones, Gaia-GOSA \\
181 & $2018-12-09.1$ & 83 & 2.22 & 3.01 & 13.2 & $V$ & C. Garcia, Gaia-GOSA \\
182 & $2018-12-10.1$ & 100 & 2.21 & 3.01 & 13.0 & $V$ & C. Garcia, Gaia-GOSA \\
183 & $2018-12-14.0$ & 54 & 2.18 & 3.01 & 11.8 & $V$ & C. Garcia, Gaia-GOSA \\
184 & $2018-12-3.3$ & 1395 & 2.28 & 3.00 & 14.8 & Rc & E. Jehin, M. Ferrais, TRAPPIST-N and -S \\
185 & $2019-1-16.2$ & 540 & 2.09 & 3.06 & 2.1 & Rc & E. Jehin, M. Ferrais, TRAPPIST-S \\
186 & $2019-1-19.2$ & 492 & 2.09 & 3.07 & 2.7 & Rc & E. Jehin, M. Ferrais, TRAPPIST-S \\
187 & $2019-1-26.3$ & 811 & 2.12 & 3.08 & 4.9 & Rc & E. Jehin, M. Ferrais, TRAPPIST-S \\
188 & $2019-2-1.2$ & 269 & 2.16 & 3.09 & 7.0 & Rc & E. Jehin, M. Ferrais, TRAPPIST-S \\
189 & $2019-1-16.1$ & 269 & 2.09 & 3.06 & 2.1 & $r$ & M. Person, T. Brothers \\
190 & $2012-12-2018-1$ & 198 & & & & $V$ & ASAS-SN \\
191 & $2015-1-2016-4$ & 16 & & & & $V$ & Gaia DR2 \\
\hline
\end{tabular}

Table A.4. Mass estimates $(\mathcal{M})$ of (704) Interamnia collected in the literature.

\begin{tabular}{|c|c|c|c|c|}
\hline \# & $\begin{array}{c}\text { Mass }(\mathcal{M}) \\
\quad(\mathrm{kg})\end{array}$ & Method & Sel. & Reference \\
\hline 1 & $(7.36 \pm 3.38) \times 10^{19}$ & DEFL & $\checkmark$ & Landgraf (1992) \\
\hline 2 & $12.3_{-12.3}^{+13.1} \times 10^{19}$ & DEFL & $x$ & Vasiliev \& Yagudina (1999) \\
\hline 3 & $(2.59 \pm 0.14) \times 10^{19}$ & DEFL & $\checkmark$ & Krasinsky et al. (2001) \\
\hline 4 & $(7.00 \pm 1.85) \times 10^{19}$ & DEFL & $\checkmark$ & Michalak (2001) \\
\hline 5 & $(1.61 \pm 0.84) \times 10^{19}$ & DEFL & $x$ & Chernetenko \& Kochetova (2002) \\
\hline 6 & $(1.61 \pm 0.84) \times 10^{19}$ & DEFL & $x$ & Kochetova (2004) \\
\hline 7 & $(7.12 \pm 0.84) \times 10^{19}$ & DEFL & $x$ & Baer \& Chesley (2008) \\
\hline 8 & $(11.30 \pm 3.18) \times 10^{19}$ & DEFL & $x$ & Ivantsov (2008) \\
\hline 9 & $(3.23 \pm 0.02) \times 10^{19}$ & EPHEM & $\checkmark$ & Fienga et al. (2009) \\
\hline 10 & $(3.69 \pm 0.37) \times 10^{19}$ & EPHEM & $\checkmark$ & Folkner et al. (2009) \\
\hline 11 & $(2.66 \pm 1.09) \times 10^{19}$ & DEFL & $\checkmark$ & Somenzi et al. (2010) \\
\hline 12 & $(3.88 \pm 0.18) \times 10^{19}$ & DEFL & $\checkmark$ & Baer et al. (2011) \\
\hline 13 & $(3.97 \pm 1.31) \times 10^{19}$ & EPHEM & $\checkmark$ & Konopliv et al. (2011) \\
\hline 14 & $(2.25 \pm 0.66) \times 10^{19}$ & DEFL & $\checkmark$ & Zielenbach (2011) \\
\hline 15 & $(3.34 \pm 0.52) \times 10^{19}$ & DEFL & $\checkmark$ & Zielenbach (2011) \\
\hline 16 & $(3.13 \pm 0.52) \times 10^{19}$ & DEFL & $\checkmark$ & Zielenbach (2011) \\
\hline 17 & $(3.88 \pm 0.75) \times 10^{19}$ & DEFL & $\checkmark$ & Zielenbach (2011) \\
\hline 18 & $(3.82 \pm 0.36) \times 10^{19}$ & EPHEM & $\checkmark$ & Fienga et al. (2011) \\
\hline 19 & $(3.82 \pm 0.47) \times 10^{19}$ & EPHEM & $\checkmark$ & Fienga et al. (2013) \\
\hline 20 & $(3.94 \pm 0.69) \times 10^{19}$ & EPHEM & $\checkmark$ & Kuchynka \& Folkner (2013) \\
\hline 21 & $(2.43 \pm 0.19) \times 10^{19}$ & EPHEM & $\checkmark$ & Pitjeva (2013) \\
\hline 22 & $(3.82 \pm 0.41) \times 10^{19}$ & EPHEM & $\checkmark$ & Fienga et al. (2014) \\
\hline 23 & $(2.72 \pm 0.12) \times 10^{19}$ & DEFL & $\checkmark$ & Goffin (2014) \\
\hline 24 & $(3.42 \pm 0.18) \times 10^{19}$ & DEFL & $\checkmark$ & Kochetova \& Chernetenko (2014) \\
\hline 25 & $(4.18 \pm 0.44) \times 10^{19}$ & EPHEM & $\checkmark$ & Viswanathan et al. (2017) \\
\hline 26 & $0.31_{-0.31}^{0.80} \times 10^{19}$ & DEFL & $x$ & Siltala \& Granvik (2017) \\
\hline \multirow[t]{2}{*}{27} & $(4.38 \pm 0.24) \times 10^{19}$ & EPHEM & $\checkmark$ & Baer \& Chesley (2017) \\
\hline & $(3.79 \pm 1.28) \times 10^{19}$ & Ave & & \\
\hline
\end{tabular}

Notes. For each, the $1 \sigma$ uncertainty, method, selection flag, and bibliographic reference are reported. The methods are DEFL: Deflection, EPHEM: Ephemeris. 